\title{
HETEROGENEOUS BELIEFS, RISK, AND LEARNING IN A SIMPLE ASSET-PRICING MODEL WITH A MARKET MAKER
}

\author{
Carl Chiarella and Xue-Zhong He \\ University of Technology, Sydney
}

This paper studies the dynamics of a simple discounted present-value asset-pricing model where agents have different risk attitudes and follow different expectation formation schemes for the price distribution. A market-maker scenario is used as the market-clearing mechanism, in contrast to the more usual Walrasian scenario. In particular, the paper concentrates on models of fundamentalists and trend followers who follow recursive geometric-decay (learning) processes (GDP) with both finite and infinite memory. The analysis depicts how the dynamics are affected by various key elements (or parameters) of the model, such as the adjustment speed of the market maker, the extrapolation rate of the trend followers, the decay rate of the GDP, the lag length used in the learning GDP, and external random factors.

Keywords: Heterogeneous Beliefs, Market Maker, Asset Pricing, Geometric-Decay Learning Process

\section{INTRODUCTION}

Research into the dynamics of financial asset prices resulting from the interaction of heterogeneous agents having different attitudes about risk and having different expectations about the future evolution of prices has flourished in recent years [e.g., Day and Huang (1990), Bullard (1994), Lux (1995, 1997, 1998), Sethi (1996), Brock and Hommes (1997a), Franke and Sethi (1998), Bullard and Duffy (1999), Franke and Nesemann (1999), and Chiarella and He (2002c)]. This literature has developed as a result of a growing dissatisfaction with (i) models of asset price dynamics based on the representative-agent paradigm, as expressed, for example, by Kirman (1992); and (ii) the extreme informational assumptions of rational expectations.

Recently, Brock and Hommes (1997b, 1998) have introduced the concept of an adaptively rational equilibrium, where agents base decisions upon predictions of

The authors are indebted to the anonymous referee for a very detailed and insightful report on an earlier version of this paper that has led to many improvements in the current version. Address correspondence to: Tony He, School of Finance and Economics, University of Technology, Sydney, P.O. Box 123 Broadway, NSW 2007, Australia; e-mail: tony.he1@uts.edu.au. 
future values of endogenous variables whose actual values are determined by equilibrium equations. A key aspect of these models is that they exhibit expectations feedback. Agents adapt their beliefs over time by choosing from different predictors or expectations functions, based upon their past performance as measured by realized profits. The resulting dynamical system is nonlinear and, as Brock and Hommes (1998) show, capable of generating the entire "zoo" of complex behavior from local stability to high-order cycles and chaos as various key parameters of the model change.

The paper builds on the work of Chiarella and $\mathrm{He}(2002 \mathrm{c})$, who extend the model of Brock and Hommes (1998) by allowing the agents to have different risk attitudes and different expectation formation schemes for both first and second moments of the price distribution. Both papers, in common with much of the earlier cited literature, use the Walrasian auctioneer scenario as the market-clearing mechanism. However, this scenario is an unsatisfactory explanation as to how market-clearing prices are arrived at in financial markets; see in this regard O'Hara (1995), who cites only one market in which market-clearing prices are arrived at via the Walrasian auctioneer scenario, and who also highlights the inadequacy of assuming this type of market-clearing mechanism. The paper presented here takes the model of Chiarella and He (2002c), but uses a market-maker scenario as the mechanism generating the market-clearing price.

In particular, the present paper investigates a simple model for the price dynamics involving only a few trading strategies. ${ }^{1}$ It is assumed that there are three types of participants in the asset market: two groups of traders-fundamentalists and trend followers - and a market maker. The market maker at the beginning of each trading period announces a price and then receives all of the buy and sell orders for the risky asset in that time period formed by agents on the basis of the announced price. The market maker hence determines the excess demand and then takes an offsetting long or short position on the risky asset so as to clear the market. The market maker announces the price for the next trading interval as a function of the excess demand in the current period. Although this scenario is still highly stylized, it does bring the analysis closer to the functioning of real markets than does the Walrasian scenario.

There is a related literature on the behavior of the market maker in securities markets, starting with Garman (1976) and continuing with Stoll (1978), Beja and Goldman (1980), Ho and Stoll (1981), Day and Huang (1990), Peck (1990), Chiarella (1992), Sethi (1996), and Farmer and Joshi (2002). Broadly speaking, these papers can be categorized into two types. The first type illustrates the tradeoffs faced by the market maker. In Ho and Stoll (1981), holding costs arise from the return risk faced by risk-averse market makers. Peck (1990) considers the opposite extreme in which a market maker faces no return risk but a significant risk of being forced to honor his or her promises as a result of adverse price changes. The second type belongs to the class of behavioral models for the price dynamics under heterogeneous expectations. Assuming linear trading rules for each type of trader, Beja and Goldman (1980) show that equilibrium is unstable when the fraction of trend 
followers is sufficiently high. Using nonlinear investment rules, Day and Huang (1990) show that this could result in chaotic price series. The Beja and Goldman model was extended by Chiarella (1992), who made the trend-following rule nonlinear. It is found that, when the fraction of trend followers is sufficiently low, the equilibrium is stable, but when it exceeds a critical value, it becomes unstable and is replaced by a limit cycle. The excess demand of each trader type oscillates as the cycle is traversed, causing sustained deviations from the equilibrium price. This model was further enhanced by Sethi (1996), who studied inventory accumulation, cash flow, and the cost of information acquisition. Farmer and Joshi (2002) use a market-maker-based method of price formation to study the price dynamics induced by several commonly used financial trading strategies to show how they amplify noise, induce structure in prices, and cause phenomena such as excess and clustered volatility.

The market maker in this paper still remains highly stylized in that he or she does not change behavior, irrespective of the size of his or her long or short position. We assume that the market maker is risk neutral, setting the price in response to excess demands from the traders, without worrying about accumulated inventory. The market framework and price formation mechanism are similar to those of Kyle (1985) and Farmer and Joshi (2002). Farmer and Joshi (2002) regard a trading strategy as a signal-processing element that uses external information and past prices as inputs and incorporates them into future prices. They show numerically how trading strategies can amplify noise, induce structure in prices, and cause phenomena such as excess and clustered volatility.

Our focus in this paper is on both the theoretical and numerical behavior of asset prices resulting from the interaction of heterogeneous investors under a (admittedly stylized) market-maker price-setting mechanism. The analysis below seeks to determine how the results of Brock and Hommes (1998) and Chiarella and $\mathrm{He}$ (2002c) are affected by this alternative market-clearing mechanism and how the price dynamics change under boundedly rational geometric-decay learning processes with finite and infinite memory. The focus is on a number of aspects of the modeling framework. First, as in Chiarella and He (2002c), traders are allowed to have differing attitudes toward risk. ${ }^{2}$ Second, the trend followers are assumed to use a geometric-decay (learning) process, based on past observations, to form their forecast rule on the price of the risky asset. With homogeneous beliefs and traders following moving-average learning processes, Balasko and Royer (1996) show that the intuition, that the larger the lag length of past information used the more stable is the Walrasian equilibrium, is essentially correct. However, with heterogeneous beliefs, the influence of the lag length becomes more complicated. For a general discussion on the dynamics of homogeneous and heterogeneous beliefs under various recursive (learning) processes, the reader is referred to Chiarella and $\mathrm{He}$ (2002a,b). In some learning analysis, such as that of Grandmont (1985), Balasko and Royer (1996) and Honkapohja and Mitra (2000), memory is bounded because the agents forget data that has occurred far enough in the past. In other analyses of learning, such as that of Evans and Honkapohja $(1994,1999)$ and Barucci $(2001)$, 
memory is unbounded or, alternatively, past data are discounted geometrically. This paper first introduces the geometric decay processes (GDP) with bounded or unbounded memory into the asset-pricing model with heterogeneous agents and then considers the learning dynamics when the trend followers use GDP. A feature of the analysis is that adaptive learning is considered in a framework that allows the fractions of the agents to vary, as in Brock and Hommes (1998) and Chiarella and $\mathrm{He}(2002 \mathrm{c})$.

The plan of the paper is as follows. Section 2 outlines the model of interacting heterogeneous agents with the market-clearing price set by a market maker, introduces the expectations function and learning mechanisms of the heterogeneous groups, and sets up the adaptive beliefs system (ABS). Section 3 considers the existence of steady states of the deterministic ABS. In Section 4, the local stability and bifurcation of the fundamental steady state are analyzed when the trend followers use the GDP with finite memory. In Section 5, various numerical tools are employed to obtain global information about the dynamics when the fundamental steady state is locally unstable, focusing in particular on how the dynamics are affected by various key elements (or parameters) of the model, such as the adjustment speed of the market maker, the extrapolation rate of the trend followers, the decay rate of the GDP, the lag length used in the learning GDP, and external random factors. A discussion on the price dynamics when the memory is infinite is included in Section 6. Section 7 concludes, and all proofs are included in the Appendix.

\section{A HETEROGENEOUS-AGENTS MODEL UNDER A MARKET MAKER}

This section sets up a standard discounted-value asset-pricing model with heterogeneous agents, which is closely related to the frameworks of Brock and Hommes (1997b, 1998) and Chiarella and He (2002c). However, as stated in the Introduction, the market-clearing price is arrived at via a market-maker scenario rather than the Walrasian scenario used in the studies just cited. We focus on the simple case in which there are three classes of participants in the asset market: two groups of traders - fundamentalists and trend chasers - and a market maker.

\subsection{Market Equilibrium Price Under a Market Maker}

Following the framework of Brock and Hommes (1998), consider an asset-pricing model with one risky asset and one risk-free asset. It is assumed that the risk-free asset is perfectly elastically supplied at gross return $R=1+r>1$ (with a fixed risk-free rate $r$ ) for each period. Let $p_{t}$ be the price (ex dividend) per share of the risky asset at time $t$ and $\left\{y_{t}\right\}$ be the stochastic dividend process of the risky asset. Then, the wealth of a typical investor at $t+1$ is given by

$$
W_{t+1}=R W_{t}+\left[p_{t+1}+y_{t+1}-R p_{t}\right] z_{t}
$$


where $W_{t}$ is wealth at time $t$ and $z_{t}$ is the number of shares of the risky asset purchased by the investor at $t$.

Denote by $F_{t}=\left\{p_{t}, p_{t-1}, \ldots ; y_{t}, y_{t-1}, \ldots\right\}$ the information set at time $t$. Let $E_{t}, V_{t}$ be the conditional expectation and variance, respectively, based on $F_{t}$, and $E_{h, t}$ and $V_{h, t}$ be the "beliefs" of investor type $h$ about the conditional expectation and variance of quantities at $t+1$. Denote by $R_{t+1}$ the excess return at $t+1$; that is,

$$
R_{t+1}=p_{t+1}+y_{t+1}-R p_{t}
$$

Then, it follows from (1) and (2) that

$$
\begin{aligned}
E_{h, t}\left(W_{t+1}\right) & =R W_{t}+E_{h, t}\left(p_{t+1}+y_{t+1}-R p_{t}\right) z_{h, t} \\
& =R W_{t}+E_{h, t}\left(R_{t+1}\right) z_{h, t} \\
V_{h, t}\left(W_{t+1}\right) & =z_{h, t}^{2} V_{h, t}\left(p_{t+1}+y_{t+1}-R p_{t}\right) \\
& =z_{h, t}^{2} V_{h, t}\left(R_{t+1}\right)
\end{aligned}
$$

where $z_{h, t}$ is the demand by agent $h$ for the risky asset.

Assume each investor type is an expected-utility maximizer, but different investors (e.g., type $h$ ) have different attitudes toward risk, characterized by the risk-aversion coefficient, $a_{h}$. Then, for type $h$, the demand $z_{h, t}$ of the risky asset is given by $^{3}$

$$
z_{h, t}=\frac{E_{h, t}\left(R_{t+1}\right)}{a_{h} V_{h, t}\left(R_{t+1}\right)} .
$$

Let $n_{h, t}$ be the fraction of investors of type $h$ at $t$ (so that $\sum_{h} n_{h, t}=1$ ). The fraction $n_{h, t}$ is updated on the basis of the fitness function, which is specified in the following discussion. Assume zero supply of outside shares. Then, the excess demand $z_{e, t}$ is given by

$$
z_{e, t} \equiv \sum_{h} n_{h, t} z_{h, t}
$$

or [using (4)]

$$
z_{e, t}=\sum_{h} n_{h, t} \frac{E_{h, t}\left[R_{t+1}\right]}{a_{h} V_{h, t}\left[R_{t+1}\right]} .
$$

To complete the model the price changes must be made explicit. The role of the market maker is to take a long (when $z_{e, t}<0$ ) or short (when $z_{e, t}>0$ ) position so as to clear the market. At the end of period $t$, after the market maker has carried out all transactions, he or she adjusts the price for the next period in the direction of the observed excess demand. Using $\mu$ to denote the corresponding speed of adjustment for each period, then, with a simple linear price adjustment process, the price would be given by

$$
p_{t+1}=p_{t}+\mu z_{e, t}
$$


which by use of (5) becomes

$$
p_{t+1}=p_{t}+\mu \sum_{h} n_{h, t} z_{h, t}
$$

Note that the market-maker behavior in this model is highly stylized. For instance, the inventory of the market maker built up as a result of the accumulation of various long and short positions is not considered. This could affect his or her behavior; for example, the market-maker price-setting role in (7) could be a function of the inventory. Allowing $\mu$ to be a function of inventory would be one way to model such behavior. Such considerations are left to future research. Future research should also seek to explore the microfoundations of the coefficient $\mu$. In the present paper, it is best thought of as a market friction, and an aim of our analysis is to understand how this friction affects the market dynamics.

\subsection{Heterogeneous Beliefs}

To get a benchmark notion of the rational-expectation fundamental solution $p_{t}^{*}$, consider the equation

$$
R p_{t}^{*}=E_{t}\left[p_{t+1}^{*}+y_{t+1}\right]
$$

where $E_{t}$ is expectation conditional on the information set $F_{t}$. In the case in which the dividend process $\left\{y_{t}\right\}$ is i.i.d., $E_{t}\left(y_{t+1}\right)=\bar{y}$ (assumed constant), the only solution satisfying the "no bubbles" condition" $\left(\lim _{t \rightarrow \infty} E p_{t} / R^{t}=0\right)$ is the constant solution $\bar{p}=\bar{y} / r$.

Regarding the heterogeneous beliefs of fundamentalists (type 1) and trend chasers (type 2) about the deviations from the fundamental solution, we assume that expectations are formed according to

$$
\begin{aligned}
& E_{1 t}\left(p_{t+1}+y_{t+1}\right)=E_{t}\left(p_{t+1}^{*}+y_{t+1}\right), \\
& E_{2 t}\left(p_{t+1}+y_{t+1}\right)=E_{t}\left(p_{t+1}^{*}+y_{t+1}\right)+d f_{t},
\end{aligned}
$$

with expected variance ${ }^{5}$

$$
V_{1 t}\left(p_{t+1}+y_{t+1}\right)=V_{2 t}\left(p_{t+1}+y_{t+1}\right)=\sigma^{2} .
$$

Here, $d>0, \sigma^{2}$ are constant, and

$$
f_{t}=\sum_{i=0}^{L-1} b_{i} p_{t-i}-p_{t}^{*}, \quad b_{i} \geq 0, \quad \sum_{i=0}^{L-1} b_{i}=1
$$

is a weighted moving-average price process with a window length $L \geq 1$. This type of process, known as a general $\boldsymbol{a}_{L}$ process is considered by Chiarella and He (2002a,b), where the dynamics of (Walrasian) steady states with both homogeneous and heterogeneous $a_{L}$ processes are studied in detail. It is found that such recursive (learning) processes can generate very rich and complicated 
dynamics. In the following discussion, three simple weighted averaging processes are considered:

(i) Moving-average process (MAP),

$$
f_{t}=\frac{1}{L} \sum_{i=0}^{L-1} p_{t-i}-p_{t}^{*}
$$

(ii) Geometric decay process (GDP),

$$
f_{t}=b\left[p_{t}+\omega p_{t-1}+\omega^{2} p_{t-2}+\cdots+\omega^{L-1} p_{t-(L-1)}\right]-p_{t}^{*},
$$

where $b=1 /\left[1+\omega+\omega^{2}+\cdots+\omega^{L-1}\right], \omega \in[0,1]$ measures the decay rate of the memory;

(iii) Limiting geometric decay process (LGDP),

$$
f_{t}=\omega f_{t-1}+(1-\omega)\left[p_{t}-p_{t}^{*}\right], \quad \omega \in[0,1) .
$$

One could interpret GDP as one of bounded memory. As $L \rightarrow \infty$, the limiting process of GDP becomes LGDP, in which memory is unbounded. For $\omega=1$, GDP leads to MAP and hence MAP can be treated as a degenerate case of GDP. Therefore, the following discussion concentrates on the geometric decay processes with both finite and infinite memory.

Under assumptions (8) and (9) with $d=0$, the fundamentalists believe that the prices will return to their fundamental values. Under the same assumption but with $d>0$, the trend chasers chase the trend of the prices by extrapolating from the weighted moving-average prices $f_{t}$ at an extrapolation rate of $d$. Here, $a_{i}(i=1,2)$ are allowed to be different to characterize the different risk attitudes of the two types of investors. Typically, as pointed by Campbell and Kyle (1993), one would expect the fundamentalists to be more risk averse than the trend chasers (that is, $a=a_{2} / a_{1}<1$ ).

\subsection{Adaptive Belief System}

Let the benchmark fundamental price be the no-bubble constant price $\bar{p}$ and let $x_{t}=p_{t}-\bar{p}$ denote the deviation of $p_{t}$ from the no-bubble fundamental price $\bar{p}$. Rewrite the excess return at period $t$ in the form

$$
R_{t}=x_{t}-R x_{t-1}+\delta_{t}
$$

where

$$
\delta_{t}=p_{t}^{*}+y_{t}-E_{t}\left[p_{t+1}^{*}+y_{t+1}\right]
$$

is a martingale difference sequence with respect to $F_{t}$; that is, $E\left[\delta_{t} \mid F_{t}\right]=0$ for all $t$. Then, under (8)-(11), one obtains

$$
E_{1 t}\left(R_{t+1}\right)=-R x_{t}, \quad E_{2 t}\left(R_{t+1}\right)=d g_{t}-R x_{t},
$$


where

$$
g_{t}=\sum_{i=0}^{L-1} b_{i} x_{t-i}, \quad b_{i} \geq 0, \quad \sum_{i=0}^{L-1} b_{i}=1 .
$$

In particular, $g_{t}=(1 / L) \sum_{i=0}^{L-1} x_{t-i}$ for MAP, $g_{t}=b\left[x_{t}+\omega x_{t-1}+\cdots+\omega^{L-1}\right.$ $x_{t-(L-1)}$ for GDP and $g_{t}=\omega g_{t-1}+(1-\omega) x_{t}$ for LGDP.

Let $\pi_{h, t}$ be the "fitness function," which is defined by the realized profits of trader type $h$ at period $t$ so that

$$
\pi_{h, t}=R_{t} z_{h, t-1}=\left[x_{t}-R x_{t-1}+\delta_{t}\right] z_{h, t-1},
$$

with

$$
z_{1, t}=\frac{-R x_{t}}{a_{1} \sigma^{2}}, \quad z_{2, t}=\frac{d g_{t}-R x_{t}}{a_{2} \sigma^{2}} .
$$

It is assumed that the fractions are updated on the basis of discrete-choice probability, as in Manski and McFadden (1981), Anderson et al. (1993), and Brock and Hommes (1997b, 1998); that is,

$$
n_{h, t}=\exp \left[\beta\left(\pi_{h, t}-C_{h}\right)\right] / Z_{t} \quad(h=1,2), \quad Z_{t}=\sum_{h} \exp \left[\beta\left(\pi_{h, t}-C_{h}\right)\right],
$$

where $C_{h} \geq 0$ is the constant cost incurred by the investors of type $h$, and $\beta(>0)$ is the intensity of choice measuring how fast agents switch between different prediction strategies. In particular, $\beta=+\infty$ means the entire mass of traders uses the strategy that has highest fitness; $\beta=0$ means that the mass of traders distributes itself evenly across the set of available strategies. Typically, it is assumed that, as informed traders, the fundamentalists incur greater costs than the chartists in the gathering of information in the formation of expectations. Here, set $C_{1}=C$ and $C_{2}=0$.

To sum up, the dynamics of the time evolution of the market are described by the ABS,

$$
\left\{\begin{array}{l}
x_{t+1}=x_{t}+\mu \sum_{h} n_{h, t} z_{h, t}, \\
n_{h, t}=\exp \left[\beta\left(\pi_{h, t}-C_{h}\right)\right] / Z_{t} \quad(h=1,2),
\end{array}\right.
$$

where

$$
\left\{\begin{aligned}
Z_{t} & =\sum_{h} \exp \left[\beta\left(\pi_{h, t}-C_{h}\right)\right], \\
\pi_{h, t} & =\left[x_{t}-R x_{t-1}+\delta_{t}\right] z_{h, t-1}, \\
z_{1, t} & =\frac{-R x_{t}}{a_{1} \sigma^{2}} \\
z_{2, t} & =\frac{d g_{t}-R x_{t}}{a_{2} \sigma^{2}}
\end{aligned}\right.
$$


Let $m_{t}=n_{1, t}-n_{2, t}$. Then, $n_{1, t}=\left(1+m_{t}\right) / 2, n_{2, t}=\left(1-m_{t}\right) / 2$ and hence the ABS (20) can be expressed as

$$
\left\{\begin{array}{l}
x_{t+1}=x_{t}+\frac{\mu}{2}\left[\left(1+m_{t}\right) z_{1, t}+\left(1-m_{t}\right) z_{2, t}\right], \\
m_{t}=\tanh \left(\frac{\beta}{2}\left[x_{t}-R x_{t-1}+\delta_{t}\right]\left[z_{1, t-1}-z_{2, t-1}\right]-\frac{\beta C}{2}\right) .
\end{array}\right.
$$

Because of the random term $\delta_{t},(22)$ is a nonlinear stochastic difference system. When $\delta_{t}=0$, the ABS (22) becomes a deterministic difference system of order $L+1$. An analysis of this deterministic system is some guide in understanding the underlying dynamics of the corresponding stochastic system.

The following sections consider the dynamic behavior of the ABS (22) under the geometric decay processes with both finite and infinite memory. As pointed out earlier, the moving-average process can be considered as a degenerate case of GDP when $\omega=1$. The existence of fundamental and nonfundamental steady states is considered first. The local stability of the fundamental steady state is analyzed. Numerical simulations are then used to verify the local stability and to demonstrate the global dynamics of the nonlinear model. In particular, the analysis depicts how the dynamics are affected by various key elements (or parameters) of the model, such as the adjustment speed of the market maker $\mu$, the extrapolation rate of the trend followers $d$, the decay rate of the GDP $\omega$, the lag length used in the learning GDP $L$, and external random factors. Some comparison with the results of Brock and Hommes (1998) and Chiarella and He (2002c) under the Walrasian scenario are also made.

\section{EXISTENCE OF STEADY STATE OF THE DETERMINISTIC ABS}

Consider the existence of the steady state of the deterministic ABS (22) when $g_{t}$ follows the general $a_{L}$ process (18). Let

$$
a=\frac{a_{2}}{a_{1}}, \quad m^{e q}=\tanh \left(-\frac{\beta C}{2}\right), \quad m^{*}=\frac{(1+a) R-d}{(1-a) R-d}, \quad \gamma=\frac{\mu}{4 a_{2} \sigma^{2}},
$$

and

$$
d^{*}=R\left[1+\frac{1+m^{e q}}{1-m^{e q}} a\right], \quad \gamma^{*}=\frac{1}{\left(1-m^{e q}\right) d^{*}} .
$$

PROPOSITION 1. Let $\delta=0$ and $x^{*}$ be the positive solution (if it exists) of

$$
\tanh \left[\frac{\beta}{2 a_{2} \sigma^{2}}(R-1)[d-(1-a) R]\left(x^{*}\right)^{2}-\frac{\beta C}{2}\right]=m^{*} .
$$

Then, the (deterministic) ABS (22) of $\left(x_{t}, m_{t}\right)$ with $g_{t}$ defined by (18) always has $a$ (fundamental) steady state $E\left(0, m^{e q}\right)$. In addition, the $A B S(22)$ has another two nonfundamental steady states $E_{ \pm}\left(x^{*}, m^{*}\right)$ if $d>d^{*}$. 
(All proofs, unless otherwise stated, are given in the Appendix.)

Proposition 1 corresponds to the existence results obtained by Brock and Hommes (1998) and Chiarella and He (2002c) under the Walrasian scenario. Therefore, in terms of the existence of fixed equilibria, both the Walrasian and market-maker scenarios lead to the same outcomes. Proposition 1 indicates that the existence of the nonfundamental steady states depends on how the trend chasers extrapolate the trend and are independent of the adjustment of the market maker. When the trend chasers extrapolate weakly (i.e., $d<d^{*}$ ), the fundamental steady state is the only steady state. However, when the trend chasers extrapolate strongly (i.e., $d>d^{*}$ ), the system generates two other nonfundamental steady states. Intuitively, the fundamental steady state is stable when the trend traders extrapolate weakly; otherwise, it becomes unstable and the nonfundamental steady states may become stable or the prices may become more volatile. The discussion in the following sections shows that this is indeed the case in general. Moreover, a broad and precise characterization of the roles of the traders and the market maker is drawn through stability and bifurcation analysis and numerical simulations.

\section{DYNAMICS OF ABS UNDER THE GEOMETRIC DECAY PROCESS WITH FINITE MEMORY}

This section considers the dynamic behavior of the ABS (22) under the geometric decay process with finite memory.

Assume that the expectations of the trend chasers follow the geometric decay process

$$
g_{t}=b\left[x_{t}+\omega x_{t-1}+\omega^{2} x_{t-2}+\cdots+\omega^{L-1} x_{t-(L-1)}\right],
$$

where $b=1 /\left[1+\omega+\omega^{2}+\cdots+\omega^{L-1}\right], \omega \in[0,1]$. Then, the deterministic ABS (22) assumes the form

$$
\left\{\begin{array}{l}
x_{t+1}=x_{t}+\frac{\mu}{2}\left[\left(1+m_{t}\right) z_{1, t}+\left(1-m_{t}\right) z_{2, t}\right] \\
m_{t+1}=\tanh \left[\frac{\beta}{2}\left(x_{t+1}-R x_{t}+\delta_{t+1}\right)\left(z_{1, t}-z_{2 . t}\right)-\frac{\beta C}{2}\right],
\end{array}\right.
$$

where

$$
z_{1, t}=\frac{-R}{a_{1} \sigma^{2}} x_{t}, \quad z_{2, t}=\frac{d g_{t}-R x_{t}}{a_{2} \sigma^{2}}
$$

\subsection{Stability Analysis}

It is generally difficult to derive sufficient conditions for the local stability of the fundamental steady state $E$ for general lag length. Some insights into the stability when the trend chasers follow the GDP may be gained by considering the simple cases when $L=2$ and 3. For $L \geq 4$, Rouche's theorem (see Appendix A.3) can be used to obtain some stability results. 
The stability analysis of the fundamental steady state can be conducted by the eigenvalues analysis of the corresponding characteristic equations. The following Proposition 2 gives the necessary and sufficient conditions for all the eigenvalues (e.g., $\left.\lambda_{i}\right)$ to satisfy $\left|\lambda_{i}\right|<1$.

PROPOSITION 2. Let $\delta_{t} \equiv 0$. Then, the fundamental steady state $E$ of (27) is locally asymptotically stable $(L A S)$ if $(d, \gamma) \in D_{L}$ for $L=1,2,3$, respectively, where

$$
D_{L}=\left\{(d, \gamma), 0<d<d^{*}, 0<\gamma<\gamma_{L}^{*}\right\}
$$

and

$$
\left\{\begin{array}{l}
\gamma_{1}^{*}=\frac{1}{\left(1-m^{e q}\right)\left(d^{*}-d\right)} \\
\gamma_{2}^{*}=\frac{1}{\left(1-m^{e q}\right)\left(d^{*}-\frac{1-\omega}{1+\omega} d\right)} \\
\gamma_{3}^{*}=\frac{1}{\left(1-m^{e q}\right)\left(d^{*}-\frac{1-\omega+\omega^{2}}{1+\omega+\omega^{2}} d\right)}
\end{array}\right.
$$

In addition, along $\gamma=\gamma_{i}^{*}(i=1,2,3)$, flip bifurcations (with -1 as one of the eigenvalues) occur and, along $d=d^{*}$, saddle-node bifurcations (with 1 as one of the eigenvalues) occur. Furthermore,

$$
D_{2} \subset D_{3} \subset D_{1}
$$

With the Walrasian market-clearing scenario in Brock and Hommes (1998) and Chiarella and $\mathrm{He}(2002 \mathrm{c})$, the local stability of the fundamental steady state is completely characterized by the extrapolation rate of the trend followers and the relative risk-aversion ratio between the fundamentalists and the trend followers, not the lag length used in the moving-average learning process for the trend followers. Also, complicated price dynamics can only be generated through saddle-nodetype bifurcations. However, Proposition 2 tells us that, with the market-maker scenario, the stability of the fundamental steady state is maintained only when the speed of the adjustment of the market maker is low and is balanced with the extrapolation rate of the trend followers. In addition, complicated price dynamics can be generated through either saddle-node or flip-type bifurcations.

In general, for any lag length, the following result on the local stability of the fundamental steady state can be derived.

PROPOSITION 3. Let $\delta_{t} \equiv 0$ and $L>1$.

(i) The fundamental steady state $E$ of the ABS (22) is LAS if $0<d<d^{*}$ and

$$
0<\gamma<\gamma_{4}^{*} \equiv \frac{1}{\left(1-m^{e q}\right)\left(d+d^{*}-2 b d\right)}
$$



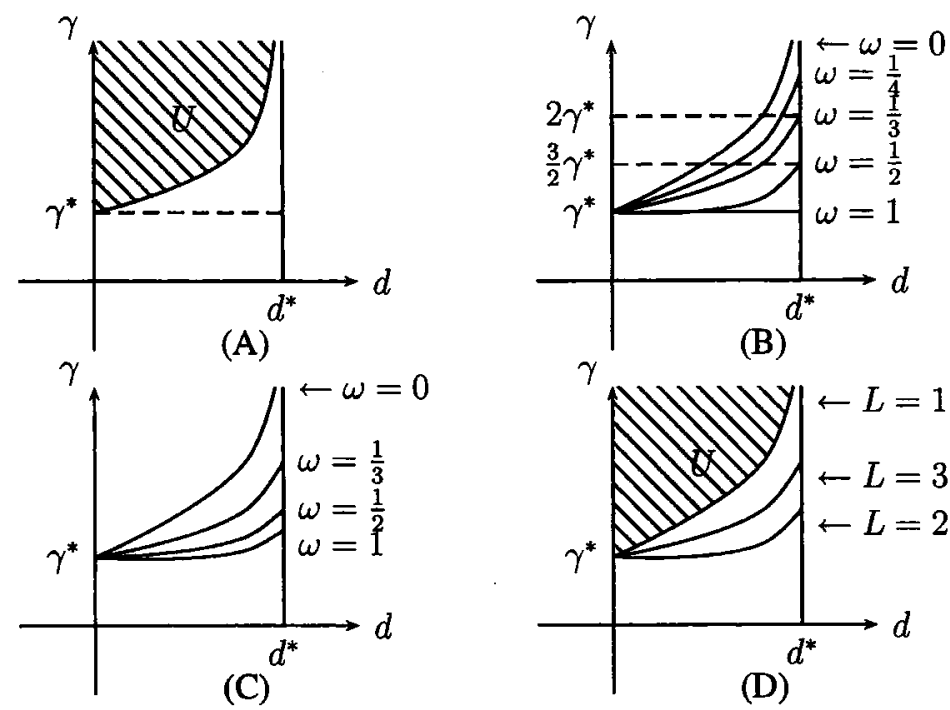

FIGURE 1. Unstable region (as indicated by $U$ ) and local stability regions (whose upper bounds are given by the various curves) of the fundamental steady state: (A) $L=1$; (B) $L=2$ with different $\omega \in[0,1]$; (C) $L=3$ with different values of $\omega$; (D) Comparison of the local stability regions for $L=1,2,3$ with fixed $\omega \in(0,1)$.

(ii) The fundamental steady state $E$ of the ABS (22) is unstable if $0<d<d^{*}$ and $\gamma>\gamma_{1}^{*}$.

Based on Propositions 2 and 3, the local unstable and stable regions of the fundamental steady state are plotted in Figure 1 for different lag length and different values of the decay rate. One can see the following:

- In terms of the stability of the fundamental steady state, it follows from $D_{2} \subset D_{3} \subset D_{1}$ (as indicated by Figure 1D) that an increase in the lag length of the GDP does not necessarily enlarge the stability region for the extrapolation rates and the adjustment speed of the market maker. In fact, $D_{1}$ is the largest stability region when $d<d^{*}$ for all the lag lengths $L \geq 2$ (as indicated by Figure $1 \mathrm{~B}$ for $L=2$ and Figure $1 \mathrm{C}$ for $L=3$ ). This contradicts a common belief that the stability regions are enlarged when agents include more historical data in forecasting rules.

- For fixed lag length $L=2,3$, a decrease in the memory decay rate $\omega$ enlarges the local stability region in terms of $d$ and $\gamma$, as indicated by Figures 1B and $1 \mathrm{C}$.

- When $\omega=0, \gamma_{L}^{*}=\gamma_{1}^{*}$ for $L=1,2,3$, implying that the stability regions $D_{L}$ for $L=2,3$ become $D_{1}$ as the decay rate of the GDP tends to zero. At $\omega=1$, $\gamma_{2}^{*}=\gamma^{*}=1 /\left[\left(1-m^{e q}\right) d^{*}\right]$ and $\gamma_{3}^{*}=\gamma_{3} \equiv 1 /\left[\left(1-m^{e q}\right)\left(d^{*}-d / 3\right)\right]$, which leads to the same stability regions when the trend chasers follow MAP with $L=2$ and 3, respectively. Therefore, the MAP can be treated as a degenerate case of the GDP. The following discussion illustrates those two special cases. 


\subsection{The Case of $L=1$}

Consider first the simplest case when either $L=1$ or $\omega=0$; that is, the trend chasers follow the forecasting rule

$$
E_{2, t}\left(p_{t+1}\right)=p_{t}^{*}+d\left[p_{t}-p_{t}^{*}\right]
$$

The trend chasers predict that prices will rise (or fall) by a constant rate $d$. Correspondingly, $g_{t}=x_{t}$ and $L=1$ for MAP or $\omega=0$ for GDP. In this case, the deterministic ABS (22) has the form

$$
\begin{aligned}
x_{t+1} & =F_{1}\left(x_{t}, m_{t}\right) \\
m_{t+1} & =\tanh \left[-\frac{\beta}{2 a_{2} \sigma^{2}}\left(F_{1}\left(x_{t}, m_{t}\right)-R x_{t}+\delta_{t+1}\right)(d-(1-a) R) x_{t}-\frac{\beta C}{2}\right],
\end{aligned}
$$

where

$$
F_{1}(x, m)=x\left[1-\frac{\mu}{2 a_{2} \sigma^{2}}(a R(1+m)+(R-d)(1-m))\right] .
$$

COROLLARY 1. The fundamental steady state $E$ of the (deterministic) ABS (29) is locally asymptotically stable (LAS) if

$$
0<d<d^{*}, \quad 0<\gamma<\gamma_{1}^{*} .
$$

In addition, along $\gamma=\gamma_{1}^{*}$, flip bifurcations occur and, along $d=d^{*}$, saddle-node bifurcations occur. Furthermore, if $0<d<R$, then $E$ is globally asymptotically stable under any one of the following conditions:

$$
\begin{aligned}
& a \geq 1,0<\gamma<1 /[2 a R] \\
& a<1, d>(1-a) R, 0<\gamma<1 /[2 a R] \\
& a<1, d<(1-a) R, 0<\gamma<1 /[2(R-d)] .
\end{aligned}
$$

Corollary 1 indicates that, with the market-maker scenario, the stability of the fundamental steady state $E$ depends on the speed of the adjustment of the market maker, the extrapolation rate of the trend chasers, and the relative risk-aversion ratio between the fundamentalists and trend chasers. Following from Corollary 1 , $E$ is LAS in a region of $(d, \gamma)$, defined by $0<\gamma<\gamma_{1}^{*}$ and $0<d<d^{*}$. Stability regions (both local and global) of the fundamental equilibrium $E$ are shown in Figure 2 in which

$$
\gamma_{1,0}=1 /[2 a R], \quad \gamma_{2,0}=1 /[2 R], \quad \gamma_{3,0}=1 /\left[\left(1-m^{e q}\right) d^{*}\right] .
$$

One can verify that both $d^{*}$ and $\gamma_{1}^{*}$ are increasing functions of $a$ and hence, as $a$ increases, the local stability region becomes larger. The condition (30) can be 


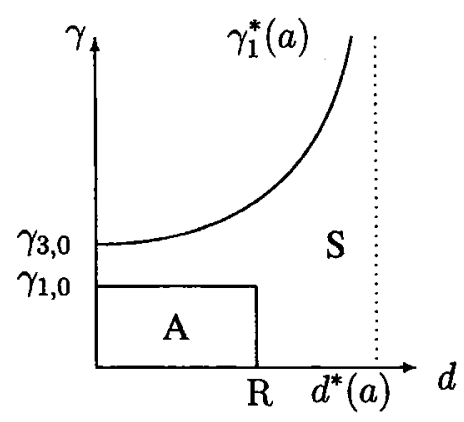

$a \geq 1$

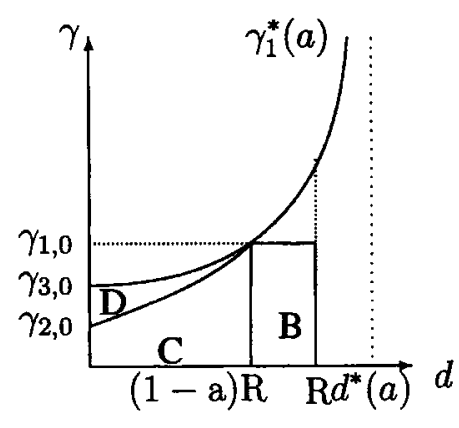

$a<1$

Figure 2. Stability regions of the fundamental steady state $S ; a \geq 1$, global stability region $A$ and local stability region $A \cup S ; a<1$, global stability region $B \cup C$ and local stability region $B \cup C \cup D$.

rewritten as $0<d<d^{*}$ and $0<\mu<\mu^{*}$, with

$$
\mu^{*}=\frac{4 a_{1} a \sigma^{2}}{a R\left(1+m^{e q}\right)+(R-d)\left(1-m^{e q}\right)} .
$$

One can verify that both $d^{*}$ and $\mu^{*}$ are increasing functions of $a$. This shows that, to maintain the (local) stability of the fundamental price, the speed of adjustment of the market maker must be proportional to the relative risk ratio $a$. Recall that a high risk ratio $a$ indicates that the trend followers are more risk averse than the fundamentalists. When the trend followers become less risk averse (relative to the fundamentalists), a lower speed of the adjustment is needed for the market maker to stabilize the fundamental price. The intuition behind this result is that an overreaction of the trend followers accompanied by a quick price adjustment (toward the fundamental price) by the market maker can in fact cause the price to be pushed away from the fundamental price.

When the trend chasers follow naive expectations, that is, $L=1$ or $\omega=0$, the global dynamics can be characterized by bifurcation diagrams and Lyapunov exponent plots. Let the risk-free rate $r=10 \%$ (i.e., $R=1.1$ ) and the cost coefficient $C$ for the fundamentalists be standardized at $C=1$. We also standardize the variance $\sigma^{2}$ and the risk-aversion coefficients $a_{1}$ and $a_{2}$ so that $a_{1} \sigma^{2}=1$ and $a=a_{2} / a_{1}$. Consider first the situation in which both fundamentalists and trend followers have the same risk-aversion coefficients $a=1$. Choose the intensity-of-choice coefficient $\beta=3.5$ and the extrapolation rate of the trend followers $d=1.2$. Assume that the speed of the adjustment $\mu$ of the market maker varies. One can verify that the fundamental steady state is $E(0,-0.9414)$ and $d^{*}=1.13322$, as defined in (24). Since $d=1.2>d^{*}$, it follows from Proposition 1 that there exist two other nonfundamental steady states $E_{ \pm}\left( \pm x^{*}, m^{*}\right)=E_{ \pm}( \pm 1.62,-0.83)$. For the parameter $\mu$, Figure $3 \mathrm{~A}$ indicates that there exist $\mu_{1} \in(0.82,0.83)$ and $\mu_{2} \in(1.06,1.07)$ such that (i) for $\mu<\mu_{1}$, all the solutions converge to either one of the two nonfundamental equilibria $E_{ \pm}$, depending on the initial values; (ii) for $\mu_{1}<\mu<\mu_{2}$, the model has either two attracting invariant circles around 

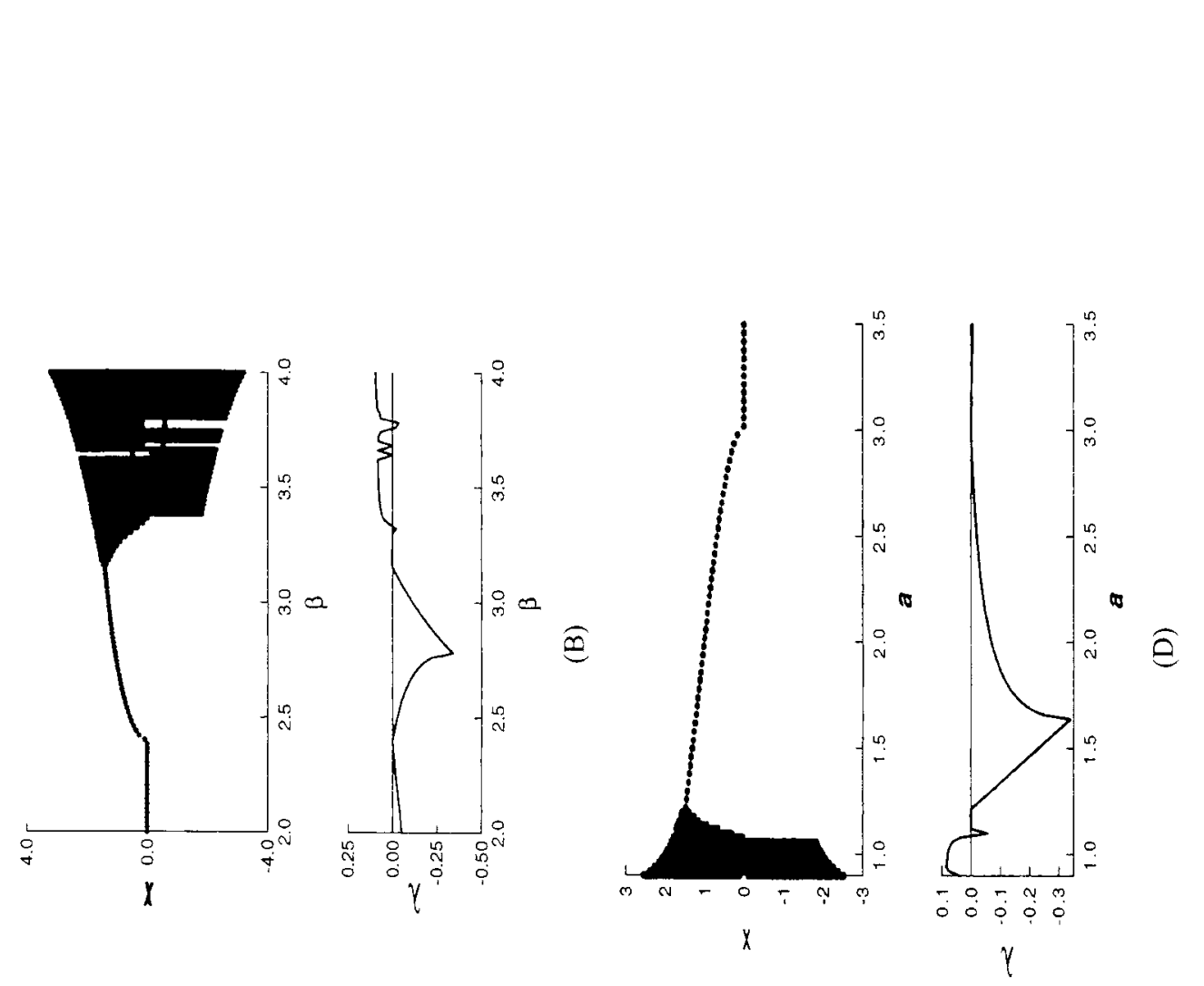

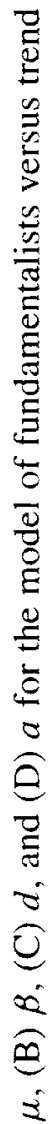
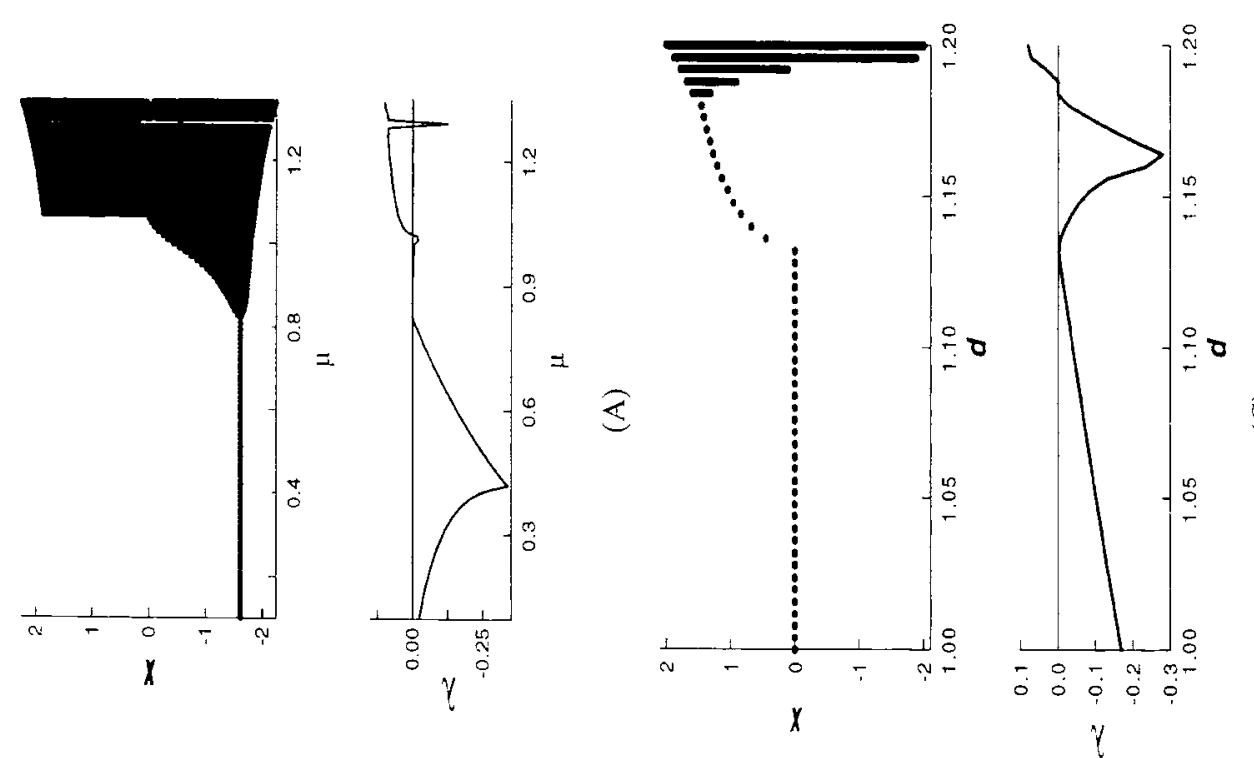

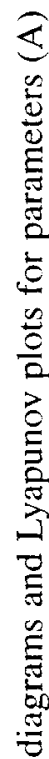

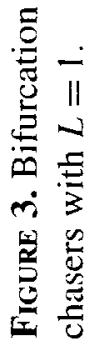


$E_{ \pm}$or strange attractors (which is indicated by the positive Lyapunov exponent in the plot); (iii) for $\mu>\mu_{2}$, the invariant circles and strange attractors cover both sides of the $x$-axis. Thus, the dynamics of the market-maker model are similar to Brock and Hommes' Walrasian model when $\mu$ is small, in that prices converge to or fluctuate around one or another of the nonfundamental equilibria. However, differences between the two market-clearing scenarios appear for large $\mu$ when fluctuations between both nonfundamental equilibria occur.

In Figure 3B, the intensity-of-choice parameter $\beta$ is selected as the bifurcation parameter. For fixed $a=1, \mu=1.2$, and $d=1.2$, the figure indicates that all the solutions converge to the fundamental steady state when $\beta$ is small $(\beta<2.4)$. As $\beta$ increases further (up to 3.14), solutions converge to either one of the nonfundamental equilibria and, for large $\beta$, the solutions display chaotic behavior, which is indicated by the positive Lyapunov exponent. Under the Walrasian scenario, the bifurcation diagrams have similar features, but the attractors for the solutions can only stay either nonpositive or nonnegative. With the market-maker scenario, the attractors can extend into both positive and negative phases. In Figure 3C, $a=1, \beta=3.5, \mu=1.2$, and $d$ is selected as the bifurcation parameter. It illustrates that all the solutions converge to the fundamental equilibrium for $d<1.132$ and to one of the nonfundamental equilibria for $1.132<d<1.18$. As $d$ increases further, the solutions either converge to some strange attractor or explode. In Figure 3D, $\beta=3.5, \mu=1.2, d=1.2$, and $a=a_{2}$ is selected as the bifurcation parameter. The figure indicates that the fundamental value is attracting when the fundamentalists are more risk averse than the trend followers [i.e., when $a(\geq 3.0)$ is large], and it becomes unstable, whereas the nonfundamental equilibria are stable as $a$ decreases. As $a$ decreases further, periodic cycles, quasi-periodic orbits, and even chaotic behavior are observed.

\subsection{The Case of the GDP with $\omega=1$ and $L=2,3$}

Recall that with $\omega=1$ the GDP degenerates to the MAP.

COROLLARY 2. The fundamental steady state $E$ of the (deterministic) ABS (22) is LAS if $d<d^{*}$ and

$$
0<\gamma<\gamma_{2} \equiv 1 /\left[\left(1-m^{e q}\right) d^{*}\right]
$$

for $L=2$, and

$$
0<\gamma<\gamma_{3} \equiv 1 /\left[\left(1-m^{e q}\right)\left(d^{*}-d / 3\right)\right]
$$

for $L=3$.

Furthermore, $\gamma=\gamma_{i}$ corresponds to a flip-type bifurcation and $d=d^{*}(i=1,2)$ corresponds to a saddle-node-type bifurcation.

Note that condition (31) for $L=2$ implies the conditions (30) and (32) for $L=1$ and $L=3$, respectively. Therefore, the local stability of $E_{1}$ for $L=2$ implies that for $L=1$ and $L=3$. It is difficult to obtain explicit conditions on local stability for 
general lag length. However, Appendix A.6 gives a sufficient condition on local stability if $\mu$ is chosen from a specific set of values.

Overall, these results suggest that, all other things being equal, the fundamental steady state of the ABS with the GDP is stabilized as the trend chasers put more weight on the most recent prices. Also, increasing the extrapolation rate $d$ up to $d^{*}$ enlarges the local stability region. However, there does not seem to be any connection between the lag length and the size of the stability region.

\section{NUMERICAL ANALYSIS OF THE DYNAMICS OF ABS UNDER THE GEOMETRIC DECAY PROCESS WITH FINITE MEMORY}

To verify the stability result established in Proposition 2 and, more importantly, to gain some insights into the price dynamics when either the market maker overadjusts the price or the trend chasers extrapolate strongly, the nonlinear adaptive beliefs system is simulated numerically.

The effect of the intensity of the choice and the relative risk-aversion (between the two traders) on the price dynamics has been studied by Brock and Hommes (1998) and Chiarella and He (2002c). The following discussion concentrates on the effect on the nonlinear dynamics of various key elements (or parameters) of the model, such as the adjustment speed of the market maker $\mu$, the extrapolation rate of the trend followers $d$, the decay rate of the GDP $\omega$, the lag length used in the GDP $L$, and external random factors (e.g., a noisy dividend process). Let

$$
R=1.1, \quad C=1.0 . \quad a_{1} \sigma^{2}=1 . \quad a_{2} \sigma^{2}=1, \quad \beta=3.5 .
$$

In this case, both the fundamentalists and the trend chasers have the same riskaversion coefficient. Then, the fundamental steady state is $E(0,-0.9414)$ and $d^{*}=1.13322$, as defined in (24). When Proposition 3 is applied, the fundamental steady state $E(0,-0.9414)$ is LAS for $d<d^{*}=1.13322$ and $\gamma<\gamma_{L}^{*}$ and unstable for either $d>d^{*}$ or $\gamma>\gamma_{L}^{*}(L=1,2,3)$. This result is confirmed numerically. The solutions become explosive when $\gamma$ crosses the flip boundary $\gamma=\gamma_{L}^{*}$, implying that weak extrapolation from the trend chasers and overadjustment from the market maker can lead to unbounded prices.

In the following discussion, ${ }^{6}$ we consider situations when the trend chasers extrapolate strongly $d=1.2>d^{*}$. In such cases, it follows from Proposition 1 that there exist two other nonfundamental steady states $E_{ \pm}\left( \pm x^{*}, m^{*}\right)=E_{ \pm}( \pm 1.62$. $-0.83)$.

\subsection{Effect of the Speed of Adjustment of the Market Maker $\mu$}

For $L=1$, Figure 4 illustrates the corresponding time series for $\mu=0.6,0.9$, and 1.2 without noise (left panel). It indicates that, when the trend chasers extrapolate strongly, a small adjustment from the market maker (e.g., $\mu=0.6$ ) leads the prices to converge to one of the nonfundamental steady states. However, as the market 


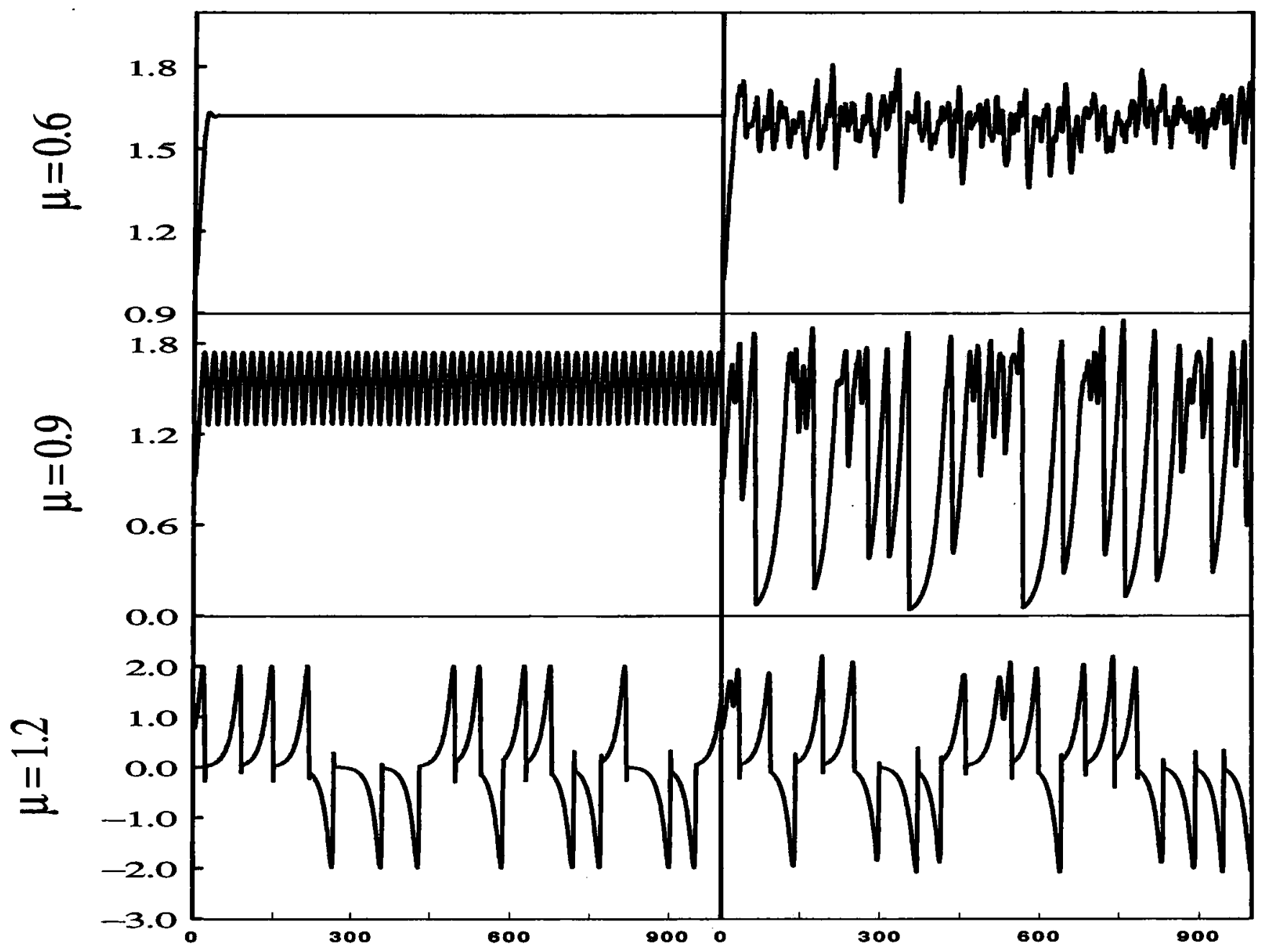



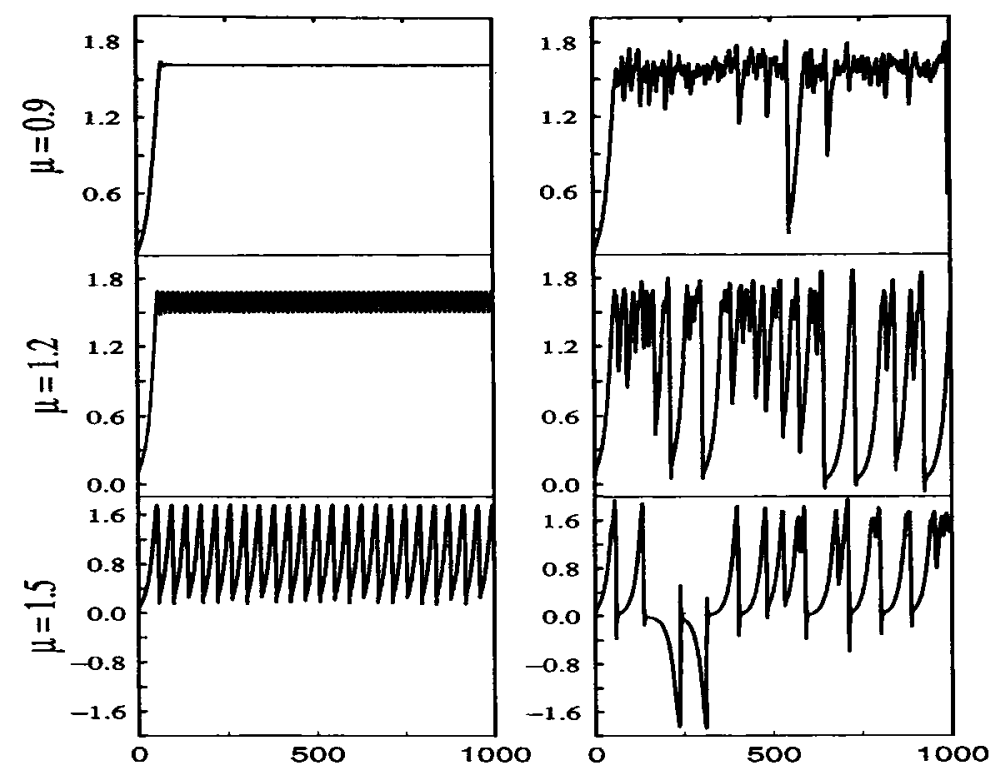

Figure 5. Time series of the GDP for $L=2$ with different values of $\mu=0.9,1.2$, and 1.5 without noise (left panel) and with noise (right panel) for $\omega=0.5$.

maker adjusts more strongly in a way such that both the forces from extrapolation of the trend chasers and the adjustment of the market maker are balanced, the prices fluctuate periodically or quasi-periodically around one of the nonfundamental steady states, as indicated when $\mu=0.9$. Large adjustment from the market maker results in the breaking of such balance, leading the prices to fluctuate among the three steady states, as indicated when $\mu=1.2$.

For $L=2$, let $\omega=0.5$ be fixed. The price dynamics are similar to the case of $L=1$, as shown in Figure 5. However, for $d=1.2>d^{*}$, an increase in $L$ (from 1 to 2) can dampen the price fluctuations in terms of the adjustment speed $\mu$ of the market maker, which is indicated by comparing the time series of $L=1$ and $L=2$ in Figures 4 and 5, respectively, for $\mu=0.9$ and $\mu=1.2$.

\subsection{Effect of the Decay Rate $\omega$ in the GDP}

To demonstrate the effect of the decay rate $\omega$ on the price dynamics when the trend chasers extrapolate strongly, for fixed $d=1.2$ and $\mu=0.95$, we selected $\omega=0.01,0.3$, and 0.95 , successively. Without noise, the price series converge to one of the nonfundamental steady states for $\omega=0.95$, as shown in Figure 6 (top plot on left panel). However, for $\omega=0.3$ and 0.01 , the price series display quasi-periodic cycles or even chaotic behavior, which can be characterized by the phase plots in the $\left(x_{t-1}, x_{t}\right)$ plane. In these cases, trajectories converge to either closed orbits or strange attractors. We have generally observed that large decay rates $\omega$ stabilize the price dynamics, whereas small decay rates $\omega$ destabilize the price dynamics, leading to quasi-periodic cycles and chaotic behavior. 

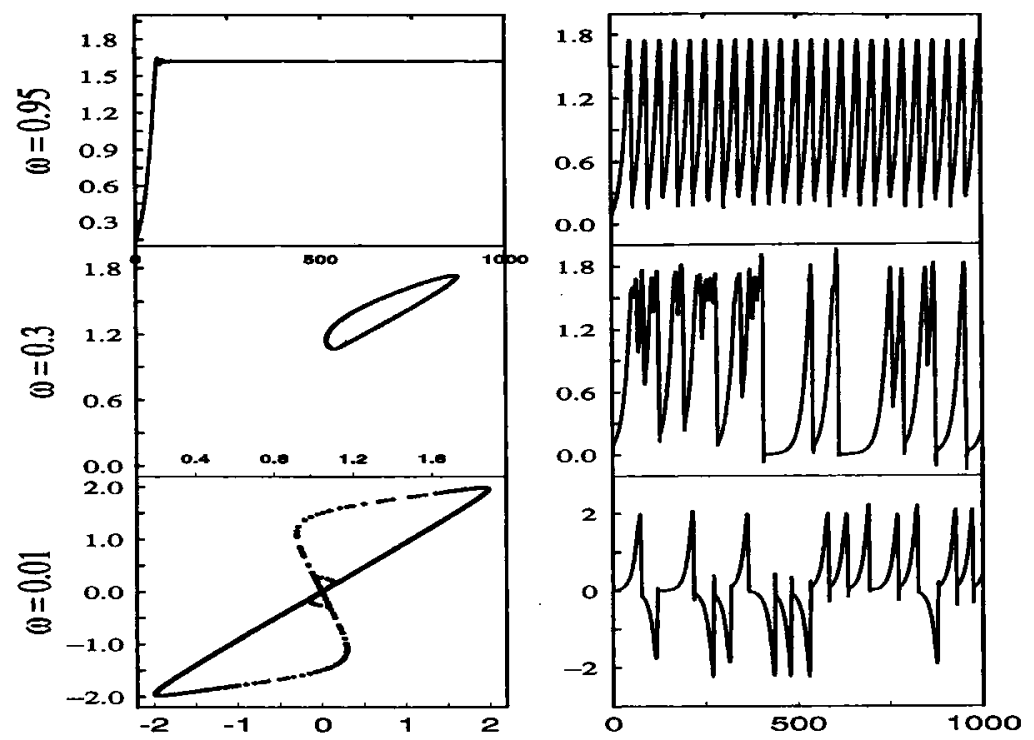

Figure 6. Time series and phase plots of the GDP for $L=2$ with different values of $\omega=0.01,0.3$, and 0.95 without noise (left panel) and the corresponding time series with noise (right panel) for $\mu=0.95$ and $d=1.2$.
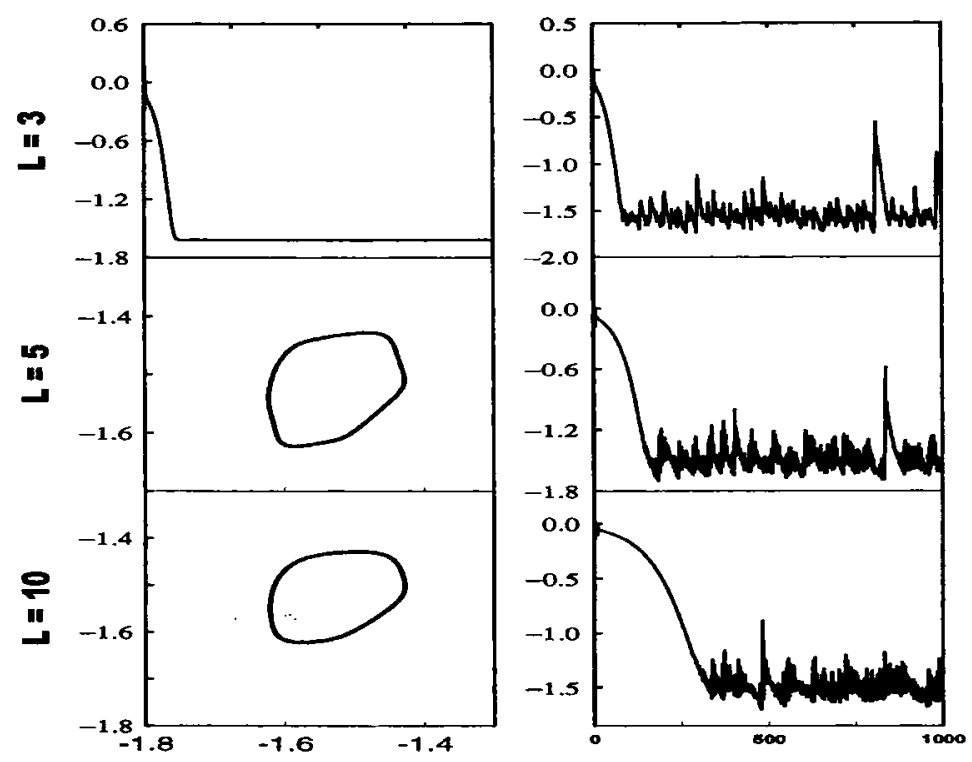

FIGURE 7. Time series and phase plots of the GDP for $L=3,5$, and 10 without noise (left panel) and with noise (right panel).

\subsection{Effect of the Lag Length $L$}

To demonstrate the effect of the leg length $L$ on the price dynamics, we select $d=1.2, \mu=1.2, \omega=0.95$, and $L=3,5$, and 10 , respectively. The corresponding time series and phase plots are shown in Figure 7. Without noise, the price series converge to one of the nonfundamental equilibria for $L=3$. However, for $L=5$ and 10 , the phase plots of the price series tend to closed orbits encircling the nonfundamental steady states. 
For the MAP, unlike the asset price model under the Walrasian scenario in Chiarella and $\mathrm{He}(2002 \mathrm{c})$, the stability conditions are much more related to the speed of the adjustment of the market maker for different lag lengths and, in general, increasing the lag length does not necessary increase the stability of the fundamental steady state. It may or may not be the case that the stability of the nonfundamental equilibria are improved as lag length increases, as indicated by the time-series plots in Figure 8, where the time series for $L=1$ is stabilized to one of the nonfundamental equilibria for $L=2$ and 3, but increasing $L$ further to 5 and 10 leads to some periodic cycles, which can be regarded as bifurcations from the nonfundamental equilibria. ${ }^{7}$ Figure 8 has been generated using the parameter set

$$
R=1.1, \quad C=1.0, \quad d=1.2, \quad a_{1} \sigma^{2}=a_{2} \sigma^{2}=1, \quad \beta=3.5, \quad \mu=1.2
$$

and $L=1,2,3,5$, and 10 .

\subsection{Effect of the Noise}

The analysis of this paper (and indeed that of most of the cited literature) has focused on the dynamics of $\mathrm{ABS}$ purely as deterministic difference equation systems. Of course, such systems will be impacted by external random factors (e.g., a noisy dividend process). The way in which these highly nonlinear ABS difference equations process external noise is an important topic for future research. Here, a very preliminary attempt is made to gauge the impact of a noisy dividend process. To this end, Figures 4-8 show the corresponding time-series plots with noise. The noise comes from a stochastic dividend process $y_{t}=\bar{y}+\epsilon_{t}$ with i.i.d. noise $\epsilon_{t}$, uniformly distributed on the interval $[-0.05,0.05]$, added to the constant dividend process $\bar{y}$. For $L=1$ and 2, prices are characterized by switching between a phase with prices close to the fundamental price and phases of upward and downward trends near the nonfundamental steady states. This result is different from the Brock-Hommes model under the Walrasian scenario, where the switching is between a phase with prices close to the fundamental price and a phase of upward (or downward) trend. In both the noise-free and noisy cases, the switching among the fundamental and nonfundamental steady states seems to be irregular. The prices fluctuate around one of the nonfundamental steady states when the market maker adjusts the prices weakly (e.g., $\mu=0.6$ for $L=1$ in Figure 4 and $\mu=0.9$ for $L=2$ in Figure 5, respectively). When such a price adjustment of the market maker is balanced by the extrapolation from the trend followers, the addition of a noisy process can cause the price to fluctuate around the nonfundamental equilibrium with occasional excursions to a neighborhood of the fundamental equilibrium. When such balanced forces disappear, the noisy process causes the prices to fluctuate among the three equilibria ( $\mu=1.2$ for $L=1$ in Figure 4 and $\mu=1.5$ for $L=2$ in Figure 5). Similar price dynamics are observed as the decay rate of the GDP $\omega$ decreases (as shown in Figure 6) and the lag length of the GDP $L$ increases. More importantly, addition of the noise process causes prices to take a longer time to come to the level near the 


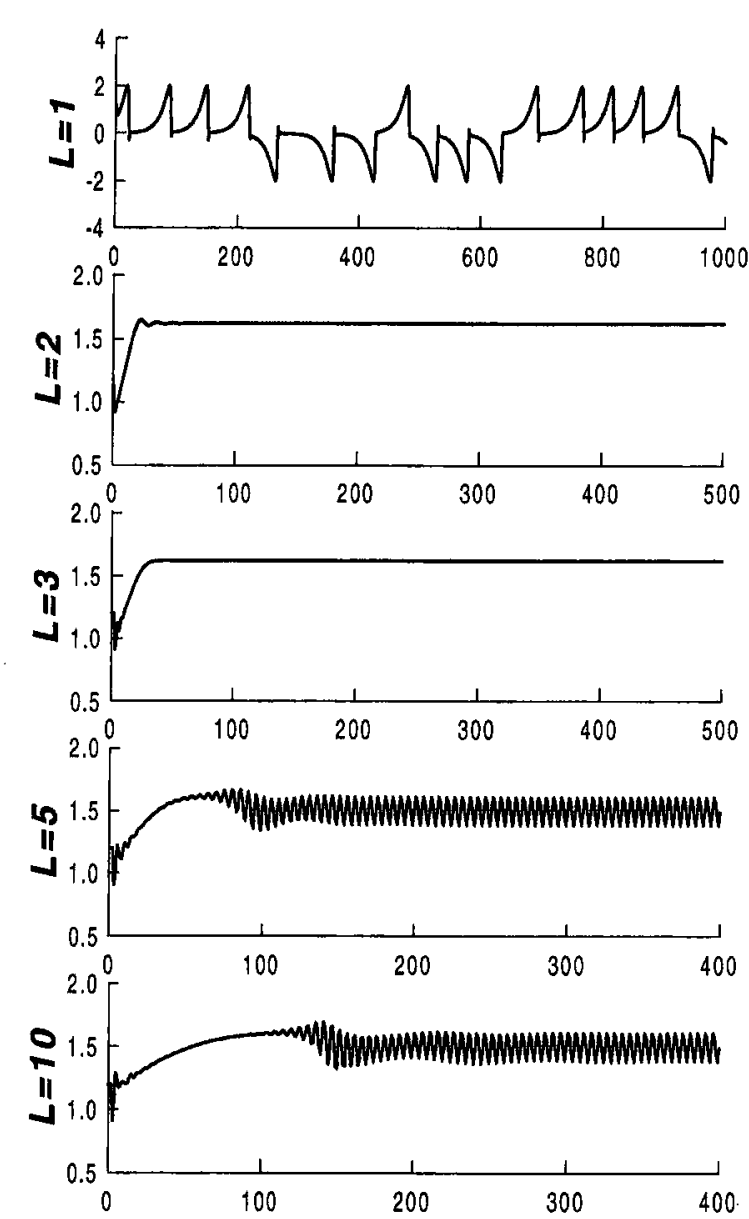

(A)
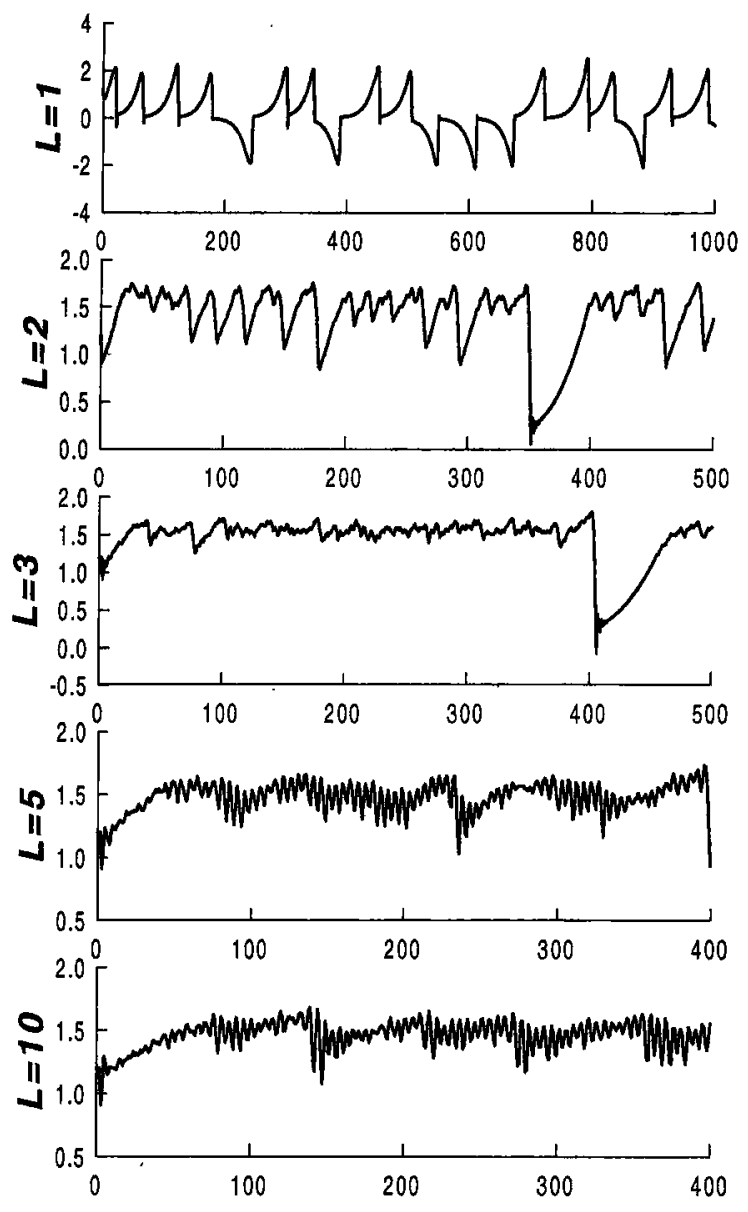

(B)

Figure 8. Time series of the MAP for different lag lengths $L=1,2,3,5$, and 10 without noise (A) and with noise (B). 
nonfundamental steady state for long lags and, more interestingly, increases the intensity of the apparent volatility clustering (see Figure 7). As might be expected, the addition of noise makes the deterministic dynamic patterns irregular. What is of interest are the occasional excursions to the other nonfundamental steady state; see, for example, the $L=3$ case in Figure 8, which suggests bimodality of the price distribution.

All of these very preliminary simulations of the noisy nonlinear adaptive belief systems are, of course, very tentative. A worthwhile future research agenda would be to conduct a Monte Carlo-type analysis of the noisy nonlinear ABS with a view to studying its statistical characteristics in terms of price distribution under the noisy dividend process.

\section{DYNAMICS OF ABS UNDER THE GEOMETRIC DECAY PROCESS WITH INFINITE MEMORY}

Considering the limit of the geometric decay process (when $L \rightarrow \infty$ ), it follows from (14) that

$$
g_{t+1}=\omega g_{t}+(1-\omega) x_{t+1}
$$

The ABS (22) then becomes

$$
\left\{\begin{array}{l}
x_{t+1}=x_{t}+\frac{\mu}{2}\left[\left(1+m_{t}\right) z_{1, t}+\left(1-m_{t}\right) z_{2, t}\right] \\
m_{t+1}=\tanh \left(\frac{\beta}{2}\left[x_{t+1}-R x_{t}+\delta_{t+1}\right]\left[z_{1, t}-z_{2, t}\right]-\frac{\beta C}{2}\right), \\
g_{t+1}=\omega g_{t}+(1-\omega) x_{t+1}
\end{array}\right.
$$

where

$$
z_{1, t}=\frac{-R x_{t}}{a_{1} \sigma^{2}}, \quad z_{2, t}=\frac{d g_{t}-R x_{t}}{a_{2} \sigma^{2}},
$$

which is a third-order nonlinear difference equation system. With regard to the existence of steady state $E\left(x^{*}, m^{*}, \bar{g}^{*}\right)$, it can be seen that the result of Proposition 1 still holds with $g^{*}=x^{*}$.

\subsection{Stability Analysis}

The eigenvalue analysis of the fundamental steady state in this case shows that the stability region is given by $D_{2}(d, \gamma)$, corresponding to the case of $L=2$ for the GDP.

PROPOSITION 4. Let $\delta_{t}=0, \omega \in(0,1)$. Then the fundamental steady state $E_{1}$ of the $A B S(34)$ is LAS if

$$
(d, \gamma) \in D(d, \gamma)=D_{2}(d, \gamma)=\left\{(d, \gamma): 0<d<d^{*}, 0<\gamma<\gamma_{2}^{*}\right\} .
$$

It is perhaps surprising that, when the memory is infinite, the stability region of the fundamental steady state is the same as the GDP with lag 

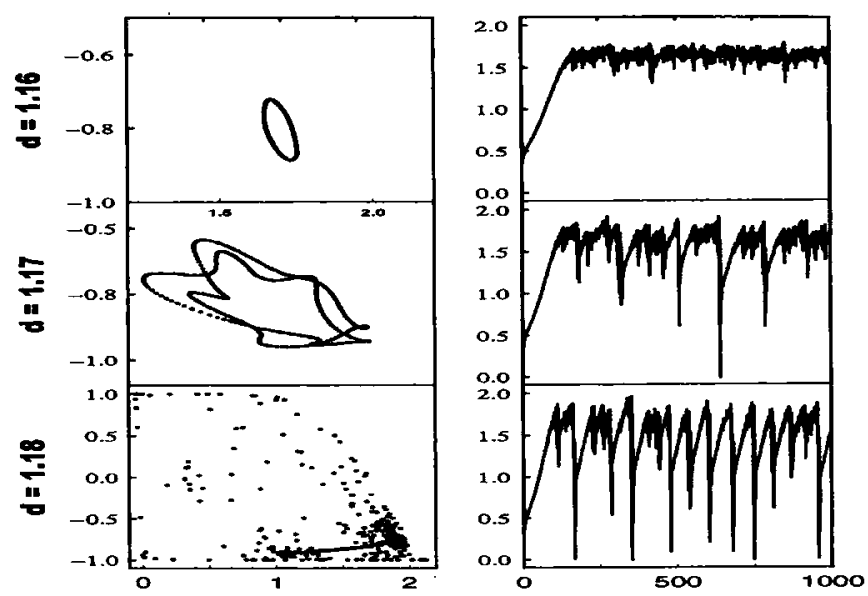

Figure 9. Limiting phase plot in $\left(x_{t}, m_{t}\right)$ plane without noise (left panel) and price time series with noise (right panel) for $\omega=0.75, a_{1}=1, a_{2}=0.5, \mu=1$, and $d=1.16,1.17$, and 1.18 .

Comparison of the stability regions of GDP with $L=1,2$, and 3, when memory is infinite leads to the smallest stability region for the fundamental steady state. Furthermore, when the trend followers extrapolate weakly, a decrease in the decay rate enlarges the stability region.

\subsection{Numerical Analysis}

Numerical simulations indicate that the prices converge to the fundamental price when both the extrapolation rate and the adjustment speed are weak (so that they are located in the local stability regions) and prices become unbounded when the prices are strongly adjusted by the market maker. Also, when the trend chasers extrapolate strongly (so that $d>d^{*}$ ), the effect of the decay rate $\omega$ on the price dynamics is similar to the case for GDP with $L \geq 2$. The following discussion considers a situation in which the trend chasers are less risk averse than the fundamentalists; that is, $a<1$. It is found that, in this case, the decay rate has a different influence on the price dynamics when the trend followers extrapolate strongly. In the following simulations, let

$$
R=1.1, \quad C=1.0, \quad a_{1}=1, \quad a_{2}=0.5, \quad \beta=3.5, \quad \sigma^{2}=1, \quad \mu=1.0 .
$$

6.2.1. Effect of the extrapolation rate $d$. Let $\omega=0.75$ be fixed. For different values of $d$, phase plots in the $\left(x_{t}, m_{t}\right)$ plane without noise and price time series with noise are plotted in Figure 9. For $d=1.16$, the $\left(x_{t}, m_{t}\right)$ trajectories converge to a closed orbit encircling one of the nonfundamental steady states. As $d$ increases, the attractors become more complicated. Addition of the noise process leads prices to fluctuate around the nonfundamental steady-state level for $d=1.16$ and, as $d$ increases further, the volatility pattern becomes more complicated, no doubt reflecting the interaction of the underlying complex deterministic dynamics and the noise. Also of interest are the not infrequent excursions to a neighborhood 

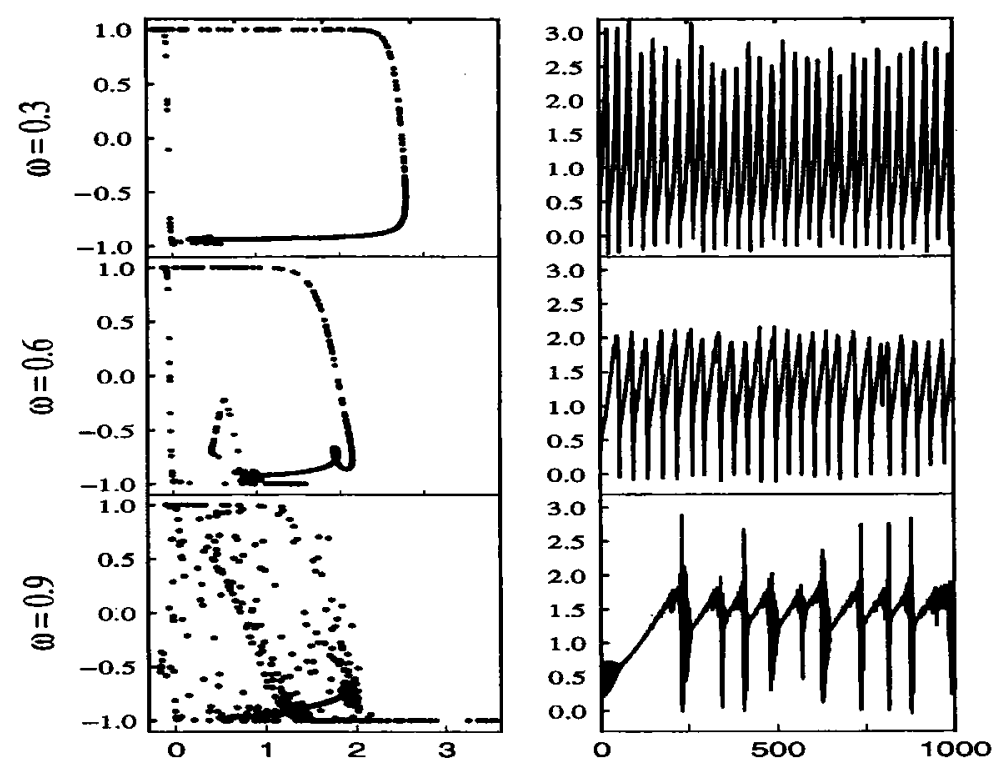

FigurE 10. Limiting phase plot in $\left(x_{t}, m_{t}\right)$ without noise (left panel) and price time series with noise (right panel) for $d=1.2, a_{1}=1, a_{2}=0.5, \mu=1$, and $\omega=0.3,0.6$, and 0.9 .

fundamental steady state. Here again, one might conjecture that the noisy nonlinear ABS is characterized by a bimodal distribution for prices.

6.2.2. Effect of the decay rate $\omega$. Let $d=1.2$ be fixed. For different values of $\omega=0.3,0.6$, and 0.9 , phase plots in the $\left(x_{t}, m_{t}\right)$ plane without noise and price time series with noise are plotted in Figure 10. For $\omega=0.3$, the time series $\left(x_{t}, m_{t}\right)$ converge to some strange attractor encircling one of the nonfundamental steady states. As $\omega$ increases, the attractors become more complicated. The addition of the noisy dividend process in this case seems to break the price pattern of the deterministic case. Now, prices fluctuate between the fundamental and one nonfundamental equilibria $(\omega=0.3$ and $\omega=0.6)$. As $\omega$ increases further $(\omega=0.9)$, one also observes high apparent volatility clustering of the prices.

\section{CONCLUSION}

Using a market-maker scenario, the present paper has incorporated risk and learning schemes into an asset-pricing model with heterogeneous beliefs. Fundamentalists and trend chasers are the main trading groups that drive the various dynamics of price changes. The importance of different learning schemes for price dynamics has been highlighted in earlier literature (and in this paper). However, the precise way in which the market clearing mechanism affects the dynamics of asset prices in models involving heterogeneity and learning has not received a great deal of attention in the literature. The current paper has sought to address this deficiency.

Under a market-maker scenario, this paper studies how the dynamics of asset prices are affected by different risk attitudes and different learning schemes of different types of investors. In particular, the paper concentrates on models of 
fundamentalists and trend followers who follow recursive geometric decay (learning) processes with both finite and infinite memory. The analysis depicts how the dynamics are affected by various key elements (or parameters) of the model, such as the adjustment speed $\mu$ of the market maker, the extrapolation rate $d$ of the trend followers, the decay rate $\omega$ of the GDP, the lag length $L$ used in the learning GDP, and external random factors. The results of the paper can be summarized as follows:

- With the Walrasian market-clearing scenario in Brock and Hommes (1998) and Chiarella and $\mathrm{He}(2002 \mathrm{c})$, the local stability of the fundamental steady state is completely characterized by the extrapolation rate of the trend followers and the relative risk-aversion ratio between the fundamentalists and the trend followers, not the lag length used in the moving-average learning process for the trend followers, and complicated price dynamics can only be generated through saddle-node-type bifurcation. In contrast, this paper has found that, with the market-maker scenario, the stability of the fundamental steady state is maintained only when the speed of the adjustment of the market maker is low and balanced with the extrapolation rate of the trend followers. Furthermore, complicated price dynamics can be generated through either saddle-node or flip-type bifurcations.

- Similar to the Walrasian scenario, in general, different lag lengths can complicate the price dynamics, but an increase in lag length may not necessarily enlarge the local stability region.

- The decay rate of the geometric-decay learning process for the trend followers has more complicated effects on the price dynamics. When the trend followers extrapolate weakly, a decrease in the decay rate for both finite and infinite memory processes enlarges the local stability region of the fundamental steady state. However, when the trend followers extrapolate strongly, the price dynamics can be stabilized by an increase in the decay rate when both the fundamentalists and trend followers have the same risk-aversion coefficients, but can be destabilized when the trend followers are less risk averse than the fundamentalists.

- When external random factors (e.g., a noisy dividend process) impinge on the dynamics, some interesting additional features are observed, in particular, excursions to other equilibria and volatility clustering. The volatility appears to be much greater than that of the external i.i.d. dividend process. However, the paper has only been able to give some brief and preliminary insights into this issue that should be topics for future research.

In summary, the study finds that the resulting dynamical behavior of the asset pricing model under the market-maker scenario is considerably enriched and has some significant differences from the Walrasian scenario. However, the interaction of external noise with the nonlinear dynamics of the model is a topic that requires more extensive research. The techniques discussed by Arnold (1998) may be useful in this regard. 
The model considered here has concentrated on the interaction of two types of investors. Of course, to mimic the price behavior observed in real financial markets, it would be preferable to allow for a larger number of investor types and/or a richer set of learning schemes from which the various investor types update their beliefs. A number of authors [see Chen and Yeh (1997, 1999), Lux and Marchesi (1999)] have developed models (similar in spirit to the one of this paper) containing many different trader types choosing from a rich set of learning schemes. Much of the analysis by those authors is necessarily based on numerical simulations. However, their models do mimic the types of price patterns observed in real financial markets, such as ARCH effects, volatility clustering, and fat tails. This paper should be seen as complementing these more numerically oriented studies by obtaining analytical results concerning local stability analysis and thus gaining some insight into the maps determining the nonlinear dynamics of this class of model.

\section{NOTES}

1. A more extensive study involving more types of agents appears in Chiarella and He (2000).

2. When different types of investors, for example, "smart-money" investors and "noise traders," are involved in the market, it is believed [see Miller (1977), Black (1986), Summers (1986), Fama and French (1988), Poterba and Summers (1988), DeLong et al. (1990), Campbell and Kyle (1993)] that the smart-money investors are more risk averse than noise traders. One message from this strand of literature is that the differing attitudes to risk of the various types of investors have an influence on the price dynamics observed.

3. Equation (4) may be derived using a mean-variance framework or by assuming that agents have a utility of wealth function $-\exp \left(-a_{h} W\right)$

4. See Hommes (2001) for further discussion of fundamental price, speculative bubbles, and rational bubbles.

5. Gaunersdorfer (2000) investigates the Brock-Hommes evolutionary asset-pricing model with time-varying variances, determined by a weighted average (with exponentially decreasing weights) of past squared returns. She shows that the results are quite similar to the Brock-Hommes results with constant variance, which is also confirmed by the analysis by Chiarella and $\mathrm{He}(2002 \mathrm{c})$. For the market-maker model, it would also be feasible to introduce a changing variance. However, numerical simulations (not reported here) also indicate that the results are quite similar to those obtained here with constant variance. Therefore, to simplify the following analysis, constant variance is assumed.

6. The generation of the simulations with noise is discussed in Section 5.4.

7. The task of conducting a stability analysis of the nonfundamental equilibria is not included in this paper due to space limitations.

\section{REFERENCES}

Anderson, S., A. de Palma, \& J. Thisse (1993) Discrete Choice Theory of Product Differentiation. Cambridge, MA: MIT Press.

Arnold, L. (1998) Random Dynamical Systems. Berlin: Springer-Verlag.

Balasko, Y. \& D. Royer (1996) Stability of competitive equilibrium with respect to recursive and learning processes. Journal of Economic Theory 68, 319-348.

Barucci, E. (2001) Fading memory learning in a class of forward-looking models with an application to hyperinflation dynamics. Economic Modelling 18, 233-252.

Beja, A. \& M. Goldman (1980) On the dynamic behavior of prices in disequilibrium. Journal of Finance 35, 235-247. 
Black, K. (1986) Noise. Journal of Finance 41, 529-543.

Brock, W. \& C. Hommes (1997a) Models of complexity in economics and finance. In C. Heij, J.M. Schumacher, B. Hanzon, \& C. Praagman (eds.), Systems Dynamics in Economic and Finance Models, pp. 3-44. New York: Wiley.

Brock, W. \& C. Hommes (1997b) A rational route to randomness. Econometrica 65, 1059-1095.

Brock, W. \& C. Hommes (1998) Heterogeneous beliefs and routes to chaos in a simple asset pricing model. Journal of Economic Dynamics and Control 22, 1235-1274.

Bullard, J. (1994) Learning equilibria. Journal of Economic Theory 64, 468-485.

Bullard, J. \& J. Duffy (1999) Using genetic algorithms to model the evolution of heterogeneous beliefs. Computational Economics 13, 41-60.

Campbell, J. \& A. Kyle (1993) Smart money, noise trading and stock price behaviour. Review of Economics and Statistics 60, 1-34.

Chen, S.-H. \& C.-H. Yeh (1997) Toward a computable approach to the efficient market hypothesis: An application of genetic programming. Journal of Economic Dynamics and Control 21, 1043-1063.

Chen, S.-H. \& C.-H. Yeh (1999) Genetic Programming in Agent-Based Artificial Markets: Simulations and Analysis. Technical report, National Chengchi University.

Chiarella, C. (1992) The dynamics of speculative behaviour. Annals of Operations Research 37, 101123.

Chiarella, C. \& X. He (2000) Heterogeneous Beliefs, Risk and Learning in a Simple Asset Pricing Model with a Market Maker. Research paper no. 35, Quantitative Finance Research Group, University of Technology, Sydney.

Chiarella, C. \& X. He (2002a) Dynamics of beliefs and learning under $\mathbf{a}_{l}$-processes-The heterogeneous case. Journal of Economic Dynamics and Control. Forthcoming.

Chiarella, C. \& X. He (2002b) Dynamics of beliefs and learning under $a_{L}$-processes-The homogeneous case. Economic Complexity, Vol. (in press) of International Symposia in Economic Theory and Econometrics, Cambridge University Press.

Chiarella, C. \& X. He (2002c) Heterogeneous beliefs, risk and learning in a simple asset pricing model. Computational Economics 19, 95-132.

Day, R. \& W. Huang (1990) Bulls, bears and market sheep. Journal of Economic Behavior and Organization 14, 299-329.

DeLong, J., A. Shleifer, L.H. Summers, \& R. Waldmann (1990) Noise trader risk in financial markets. Journal of Political Economy 98, 703-738.

Evans, G. \& S. Honkapohja (1994) On the local stability of sunspot equilibria under adaptive learning rules. Journal of Economic Theory 64, 142-161.

Evans, G. \& S. Honkapohja (1999) Learning dynamics. In J.B. Taylor \& M. Woodford (eds.) Handbook of Macroeconomics, pp. 449-542. Amsterdam: Elsevier.

Fama, E. \& K. French (1988) Permanent and temporary components of stock prices. Journal of Political Economy 96, 246-273.

Farmer, J.D. \& S. Joshi (2002) The price dynamics of common trading strategies. Journal of Economic Behavior and Organization 49, 149-171.

Franke, R. \& T. Nesemann (1999) Two destabilizing strategies may be jointly stabilizing. Joumal of Economics 69, 1-18.

Franke, R. \& R. Sethi (1998) Cautious trend-seeking and complex asset price dynamics. Research in Economics 52, 61-79.

Garman, M. (1976) Market microstructure. Journal of Financial Economics 3, 257-275.

Gaunersdorfer, A. (2000) Endogenous fluctuations in a simple asset pricing model with heterogeneous agents. Journal of Economic Dynamics and Control 24, 799-831.

Grandmont, J.-M. (1985) On endogenous competitive business cycles. Econometrica 53, 995-1045.

Ho, T. \& H. Stoll (1981) Optimal dealer pricing under transactions and return uncertainty. Journal of Financial Economics 9, 47-73.

Hommes, C. (2001) Financial markets as nonlinear adaptive evolutionary systems. Quantitative Finance 1, 149-167. 
Honkapohja, S. \& K. Mitra (2000) Learning with Bounded Memory in Stochastic Models. Working paper, University of Helsinki and University of York.

Kirman, A. (1992) Whom or what does the representative agent represent? Journal of Economic Perspectives 6, 117-136.

Kyle, A. (1985) Continuous auctions and insider trading. Econometrica 53, 1315-1335.

Lux, T. (1995) Herd behaviour, bubbles and crashes. Economic Journal 105, 881-896.

Lux, T. (1997) Time variation of second moments from a noise trader/infection model. Journal of Economic Dynamics and Control 22, 1-38.

Lux, T. (1998) The socio-economic dynamics of speculative markets: Interacting agents, chaos, and the fat tails of return distributions. Journal of Economic Behavior and Organization 33, 143-165.

Lux, T. \& M. Marchesi (1999) Scaling and criticality in a stochastic multi-agent model of a financial markets. Nature 397, 498-500.

Manski, C. \& D. McFadden (1981) Structural Analysis of Discrete Data with Econometric Applications. Cambridge, MA: MIT Press.

Miller, E. (1977) Risk, uncertainity, and divergence of opinion. Journal of Finance 32, 1151-1168.

O'Hara, M. (1995) Market Microstructure Theory. Cambridge, MA: Blackwell.

Peck, J. (1990) Liquidity without money: A general equilibrium model of market microstructure. Journal of Financial Intermediation 1, 80-103.

Poterba, J. \& L. Summers (1988) Mean reversion in stock prices: Evidence and implications. Journal of Financial Economics 22, 27-60.

Sethi, R. (1996) Endogenous regime switching in speculative markets. Structural Change and Economic Dynamics 7, 99-118.

Stoll, H. (1978) The supply of dealers services in securities markets. Journal of Finance 33, 1133-1151.

Summers, L. (1986) Does the stock market rationally reflect fundamental values. Joumal of Finance $41,591-601$.

\section{APPENDIX A}

\section{A.1. PROOF OF PROPOSITION 1}

Let $\bar{x}, \bar{m}$ be the steady state of (22) and $A_{1}=R / a_{1}, A_{2}=(R-d) / a_{2}$. Then,

$$
\begin{aligned}
& \bar{x}=\bar{x}+\frac{\mu}{2}\left[(1+\bar{m}) \bar{z}_{1}+(1-\bar{m}) \bar{z}_{2}\right] \\
& \bar{m}=\tanh \left(\frac{\beta(1-R)}{2} \bar{x}\left[\bar{z}_{1}-\bar{z}_{2}\right]-\frac{\beta C}{2}\right)
\end{aligned}
$$

with $\bar{z}_{1}=-R \bar{x} /\left(a_{1} \sigma^{2}\right)=-A_{1} \bar{x} / \sigma^{2}$ and $\bar{z}_{2}=(d-R) \bar{x} /\left(a_{2} \sigma^{2}\right)=-A_{2} \bar{x} / \sigma^{2}$. Hence the solution of (A.1) satisfies

$$
\begin{gathered}
{\left[A_{1}(1+\bar{m})+A_{2}(1-\bar{m})\right] \bar{x}=0} \\
\bar{m}=\tanh \left[\frac{\beta}{2 \sigma^{2}}(R-1)\left(A_{1}-A_{2}\right) \bar{x}^{2}-\frac{\beta}{2} C\right] .
\end{gathered}
$$


The first equation implies $\bar{x}=0$, or $\bar{m}=\left(A_{2}+A_{1}\right) /\left(A_{2}-A_{1}\right) \equiv m^{*} \cdot \bar{x}=0$ implies $\bar{m}=m^{e q}$ which leads to the existence of $E$. Assume $\bar{x} \neq 0$; then, $\bar{m}=m^{*}$.

Note that $A_{1}>0$. If $d<R$, then $A_{2}>0$ and hence $m^{*}>1$ for $A_{2}>A_{1}$ and $m^{*}<-1$ for $A_{2}<A_{1}$. Therefore, there is no other solution when $d<R$. When $d>R$, then $A_{2}<0$ and one can verify that $\bar{m} \in(-1,1)$. If $A_{2}<-A_{1}$, that is, $d>(1+a) R$, then $0<m^{*}<1$ and hence equation (A.2) always has a solution for some $\pm \bar{x}$. This indicates the existence of $E_{ \pm}$. Now, if $A_{2}>-A_{1}$-that is, $d<(1+a) R$-then-1<m* $<0$. Then, equation (A.2) has a solution for some $\pm \bar{x}$ if and only if $m^{e q}<m^{*}$, which is equivalent to $d>d^{*}$. Note that $d^{*}<(1+a) R$. Hence, in both cases, equation (A.2) has a solution for some $\pm \bar{x}$. This completes the proof.

\section{A.2. PROOF OF PROPOSITION 2}

The system (27) is equivalent to an $L+1$ order difference equation

$$
x_{t+1}=F\left(x_{t}, x_{t-1}, \ldots, x_{t-(L-1)}, x_{t-L}\right),
$$

where

$$
\left\{\begin{aligned}
F\left(x_{t}, x_{t-1}, \ldots, x_{t-L}\right) & =x_{t}+\frac{\mu}{2}\left[\left(1+m_{t}\right) \frac{\left(-R x_{t}\right)}{a_{1} \sigma^{2}}+\left(1-m_{t}\right) \frac{d g_{t}-R x_{t}}{a_{2} \sigma^{2}}\right] \\
g_{t} & =b_{1} x_{t}+b_{2} x_{t-1}+\cdots+b_{L} x_{t-(L-1)}
\end{aligned}\right.
$$

and

$$
m_{t}=\tanh \left[\frac{\beta}{2}\left(x_{t}-R y_{t}\right)\left(\frac{-R y_{t}}{a_{1} \sigma^{2}}-\frac{d g_{t}-R y_{t}}{a_{2} \sigma^{2}}\right)-\frac{\beta C}{2}\right] .
$$

At the steady state $E, x^{*}=0$. Evaluate the partial derivatives of $F$ at $\left(x_{t}, x_{t-1}, \ldots, x_{t-L}\right)=$ $\left(x^{*}, x^{*}, \ldots, x^{*}\right)=(0,0, \ldots, 0)$ :

$$
\begin{aligned}
\frac{\partial F}{\partial x_{t}} & =1+\frac{\mu}{2 a_{2} \sigma^{2}}\left(1-m^{e q}\right)\left[b_{1} d-R\left(1+a \frac{1+m^{e q}}{1-m^{e q}}\right)\right] \\
& =1+2 \gamma\left(1-m^{e q}\right)\left[b_{1} d-d^{*}\right] \equiv A, \\
\frac{\partial F}{\partial x_{t-i}} & =\frac{\mu}{2 a_{2} \sigma^{2}}\left(1-m^{e q}\right) d b_{i+1}=2 \gamma\left(1-m^{e q}\right) d b_{i+1} \equiv B_{i+1}, \\
& \quad(i=1,2, \ldots, L-1), \\
\frac{\partial F}{\partial x_{t-L}} & =0 .
\end{aligned}
$$

The characteristic equation of the fundamental steady state $E$ is given by

$$
\lambda\left[\lambda^{L}-A \lambda^{L-1}-B_{2} \lambda^{L-2}-\cdots-B_{L}\right]=0
$$

Thus, one eigenvalue is zero and the remaining $L$ eigenvalues are given by the solutions to

$$
\Gamma_{L}(\lambda) \equiv \lambda^{L}-A \lambda^{L-1}-B_{2} \lambda^{L-2}-\cdots-B_{L}=0 .
$$


- For $L=1, b_{1}=1$, and

$$
\Gamma_{1}(\lambda) \equiv \lambda-A=0,
$$

one obtains $|\lambda|<1$ iff $|A|<1$; that is,

$$
-1<1+2 \gamma\left(1-m^{e q}\right)\left[d-d^{*}\right]<1 .
$$

That is,

$$
0<\gamma\left(1-m^{e q}\right)\left(d^{*}-d\right)<1,
$$

which is equivalent to

$$
0<d<d^{*}, \quad 0<\gamma<\frac{1}{\left(1-m^{e q}\right)\left(d^{*}-d\right)}=\gamma_{1}^{*} .
$$

Also, $\lambda=1$ along $d=d^{*}$ and $\lambda=-1$ along $\gamma=\gamma_{1}^{*}$.

- For $L=2, b_{1}=1 /(1+\omega), b_{2}=\omega /(1+\omega)$. Hence, $B=2 \gamma\left(1-m^{e q}\right) d \omega /(1+\omega)$ and

$$
\Gamma_{2}(\lambda) \equiv \lambda^{2}-A \lambda-B=0 .
$$

Using Jury's test, $\left|\lambda_{i}\right|<1$ iff $\Gamma_{2}(1)>0$ and $(-1)^{2} \Gamma_{2}(-1)>0$; that is,

$$
\begin{aligned}
& 1-A-B>0, \\
& 1+A-B>0 .
\end{aligned}
$$

One can verify that these conditions are equivalent to

$$
0<d<d^{*}, \quad 0<\gamma<\gamma_{2}^{*} .
$$

Also, $\lambda=1$ is one of the eigenvalues along $d=d^{*}$ and $\lambda=-1$ is one of the eigenvalues along $\gamma=\gamma_{2}^{*}$.

- For $L=3, b_{1}=1 /\left(1+\omega+\omega^{2}\right), b_{2}=\omega /\left(1+\omega+\omega^{2}\right), b_{3}=\omega^{2} /\left(1+\omega+\omega^{2}\right)$, and

$$
\begin{aligned}
& \left\{\begin{array}{c}
B_{2}=2 \gamma\left(1-m^{e q}\right) \frac{\omega d}{1+\omega+\omega^{2}} \\
B_{3}=2 \gamma\left(1-m^{e q}\right) \frac{\omega^{2} d}{1+\omega+\omega^{2}} .
\end{array}\right. \\
& \Gamma_{3}(\lambda) \equiv \lambda^{3}-A \lambda^{2}-B_{2} \lambda-B_{3}=0 .
\end{aligned}
$$

Using Jury's test, $\left|\lambda_{i}\right|<1$ iff $-B_{2}<3$ and

$$
\begin{aligned}
& \pi_{1} \equiv 1-A-B_{2}-B_{3}>0, \\
& \pi_{2} \equiv 1+A-B_{2}+B_{3}>0, \\
& \pi_{3} \equiv 1+B_{2}+A B_{3}-B_{3}^{2}>0 .
\end{aligned}
$$

Furthermore, $\lambda=1(-1)$ is one of the eigenvalues along $\pi_{1}=0\left(\pi_{2}=0\right)$. One can verify that $\pi_{1}>0$ if $d<d^{*}, \pi_{2}>0$ if $0<\gamma<\gamma_{3}^{*}$ and $\pi_{1}>0, \pi_{2}>0$ imply $\pi_{3}>0$. Note that

$$
\frac{1-\omega}{1+\omega}<\frac{1-\omega+\omega^{2}}{1+\omega+\omega^{2}}<1
$$


which implies that

$$
\gamma_{2}^{*}<\gamma_{3}^{*}<\gamma_{1}^{*}
$$

Therefore

$$
D_{2} \subset D_{3} \subset D_{1}
$$

\section{A.3. ROUCHE'S PROPOSITION}

If the complex functions $f(z)$ and $g(z)$ are analytic inside and on a simple closed curve $\gamma$ and if $|g(z)|<|f(z)|$ on $\gamma$, then $f(z)$ and $f(z)+g(z)$ have the same number of zeros inside $\gamma$.

\section{A.4. PROOF OF PROPOSITION 3}

(i) To apply Rouche's Proposition, take $\gamma$ to be the unit circle $|z|=1$, the function $f(z)=z^{L}$, while $g(z)=-A z^{L-1}-B_{2} z^{L-2}-\cdots-B_{2}$. Then, on $\gamma:|z|=1,|f(z)|=1$ and $|g(z)| \leq|A|+\sum_{i=2}^{L}\left|B_{i}\right|$. Note that

$$
\sum_{i=2}^{L}\left|B_{i}\right|=2 \gamma\left(1-m^{e q}\right) d\left(1-b_{1}\right), \quad b_{1}=b .
$$

It can be verified that

$$
|A|+\sum_{i=2}^{L}\left|B_{i}\right|<1
$$

is equivalent to the conditions $d<d^{*}$ and (28) of Proposition 3, leading to $|g(z)|<|f(z)|$ on $\gamma$. By Rouche's Proposition, it follows that $p(z)=f(z)+g(z)$ and $f(z)=z^{L}$ have the same number of zeros inside the unit circle. Since $f(z)=z^{L}=0$ has $L$ repeated zeros $z=0$ inside the unit circle, all the zeros of $p(\lambda)$ lie inside the unit circle and, consequently, $E$ is locally asymptotically stable.

(ii) To apply Rouche's Proposition, take $\gamma$ to be the unit circle $|z|=1$. The function $f(z)=\left(B_{1}-A\right) z^{L-1}$, while $g(z)=z^{L}-B_{1} z^{L-1}-B_{2} z^{L-2}-\cdots-B_{2}$ with $B_{1}=b$. Then, on $\gamma:|z|=1,|f(z)|=|b-A|$ and $|g(z)| \leq 1+\sum_{i=1}^{L}\left|B_{i}\right|=1+2 \gamma\left(1-m^{e q}\right) d$. Under the condition $0<d<d^{*}$ and $\gamma>\gamma_{1}^{*}$, one can verify that $|g(z)| \leq 1+\sum_{i=1}^{L}\left|B_{i}\right| \leq$ $|b-A|=|f(z)|$ on $\gamma$. It follows from Rouche's Proposition that $p(z)=f(z)+g(z)$ and $f(z)=(b-A) z^{L-1}$ have the same number of zeros inside the unit circle. Since $f(z)=(b-A) z^{L-1}=0$ has $L-1$ repeated zeros $z=0$ inside the unit circle, there are only $L-1$ zeros of $p(\lambda)$ lying inside the unit circle, which implies that there is one zero satisfying $|z| \geq 1$. Consequently, $E$ is unstable.

\section{A.5. PROOF OF COROLLARY 1}

In the case of $L=1$, the local stability result follows from the Proposition 2 .

Now assume $0<d<R$ and let

$$
h(m)=1-\frac{\mu}{2 a_{2} \sigma^{2}}[a R(1+m)+(R-d)(1-m)] .
$$


Then it follows from $-1<m<1$ and

$$
h^{\prime}(m)=-\frac{\mu}{2 a_{2} \sigma^{2}}[(a-1) R+d]
$$

that if $d>(1-a) R$, then $h(1)<h(m)<h(-1)$, and if $d<(1-a) R$, then $h(-1)<h(m)<$ $h(1)$. Note that $h(1)=1-4 \gamma a R$ and $h(-1)=1-4 \gamma(R-d)$. From the first equation of (29), one can see that $E$ is globally asymptotically stable iff $-1<h(1), h(-1)<1$ for $d>$ $(1-a) R$ and $-1<h(-1), h(1)<1$ for $d<(1-a) R$, which can be verified under either one of the conditions in Corollary 1.

\section{A.6. A STABILITY RESULT FOR THE MAP WITH ANY LAG LENGTH}

It is generally difficult to obtain explicit sufficient conditions on the local stability of the GDP for any lag length. However, when $\omega=1$, the GDP leads to the MAP and $B_{i}=B \equiv 1+2 \gamma\left(1-m^{e q}\right)\left[d / L-d^{*}\right]$. In this case, the following Lemma from Chiarella and $\mathrm{He}(2002 \mathrm{c})$ can be used to obtain a stability result.

LEMMA A.1. Let

$$
p(\lambda)=\lambda^{L}+\gamma \lambda^{L-1}+\gamma \lambda^{L-2}+\cdots+\gamma \lambda+\gamma .
$$

Then, zeros of $p(\lambda)$ lie inside the unit circle if and only if

$$
-\frac{1}{L}<\gamma<1
$$

If $\mu$ is selected such that $A=B$, then Lemma A.1 leads to the following stability result for $L \geq 1$.

COROLLARY A.1. Assume that

$$
\gamma=1 /\left[2\left(1-m^{e q}\right) d^{*}\right]
$$

Then, $E$ of system (22) is LAS if $0<d<d^{*}$.

Under the Walrasian scenario, the same sufficient condition $d<d^{*}$ on the local stability of the Walrasian fixed equilibrium $E_{1}$ is obtained by Chiarella and $\mathrm{He}(2002 \mathrm{c})$. Note that the condition $d<d^{*}$ is independent of the lag length $L$. Under the market-maker scenario, Corollary A.1 indicates that, when the speed of the adjustment of the market maker is selected as in (A.4), the stability condition is independent of the lag length $L$. Let

$$
a^{*}=\left[\frac{d^{*}}{R}-1\right] \frac{1-m^{e q}}{1+m^{e q}} .
$$

Hence, under condition (A.4), $E_{1}$ is LAS for $a>a^{*}$. In the special case that the information costs and the risk-aversion coefficients are the same (i.e., $C=0$ and $a_{1}=a_{2}$ ), (A.4) and $d<d^{*}$ lead to

$$
\gamma=\frac{\mu}{4 a_{2} \sigma^{2}}=\frac{4}{R}, \quad d<2 R .
$$

This indicates that, in this case, the fundamental steady state is LAS if the trend chasers do not extrapolate strongly $(0<d<2 R)$ and the speed of the adjustment of the market maker 
is proportional to the risk-aversion coefficient $\left(a_{2}\right)$ with the product of the variance $\left(\sigma^{2}\right)$ and the discount rate of return $(1 / R)$ as the coefficient.

\section{A.7. PROOF OF PROPOSITION 4}

The ABS has the following form:

$$
\left\{\begin{array}{l}
x_{t+1}=F\left(x_{t}, y_{t}, g_{t}\right) \\
y_{t+1}=x_{t} \\
g_{t+1}=G\left(x_{t}, y_{t}, g_{t}\right)
\end{array}\right.
$$

where

$$
\left\{\begin{array}{l}
F\left(x_{t}, y_{t}, g_{t}\right)=x_{t}+\frac{\mu}{2}\left[\left(1+m_{t}\right) \frac{-R x_{t}}{a_{1} \sigma^{2}}+\left(1-m_{t}\right) \frac{d g_{t}-R x_{t}}{a_{2} \sigma^{2}}\right] \\
G\left(x_{t}, y_{t}, g_{t}\right)=\omega g_{t}+(1-\omega) F\left(x_{t}, y_{t}, g_{t}\right)
\end{array}\right.
$$

and

$$
m_{t}=\tanh \left[\frac{\beta}{2}\left(x_{t}-R y_{t}\right)\left(\frac{-R y_{t}}{a_{1} \sigma^{2}}-\frac{d g_{t}-R y_{t}}{a_{2} \sigma^{2}}\right)-\frac{\beta C}{2}\right] .
$$

One can verify that, at the steady state $\left(x^{*}, y^{*}, g^{*}\right)=(0,0,0)$,

$$
\begin{cases}\frac{\partial F}{\partial x_{t}}=A=1-2 \gamma\left(1-m^{e q}\right) d^{*}, & \frac{\partial G}{\partial x_{t}}=(1-\omega) A \\ \frac{\partial F}{\partial y_{t}}=0, & \frac{\partial G}{\partial y_{t}}=0 \\ \frac{\partial F}{\partial g_{t}}=B=2 \gamma d\left(1-m^{e q}\right), & \frac{\partial G}{\partial g_{t}} \equiv C=\omega+(1-\omega) B .\end{cases}
$$

Hence, the characteristic equation is

$$
\Gamma_{3}(\lambda) \equiv \lambda[(\lambda-A)(\lambda-C)-A B(1-\omega)]=0 .
$$

Note that

$$
D \equiv A C-A B(1-\omega)=\omega A
$$

Then,

$$
\Gamma_{3}(\lambda)=\lambda\left[\lambda^{2}-(A+C) \lambda+\omega A\right]=0
$$

and $\left|\lambda_{i}\right|<1$ iff $0<d<d^{*}, 0<\gamma<\gamma_{2}^{*}$. Furthermore, $\lambda=1(\lambda=-1)$ is one of the eigenvalues along $d=d^{*}\left(\gamma=\gamma_{2}^{*}\right)$. 
Home $>$ Journals $>$ Macroeconomic Dynamics

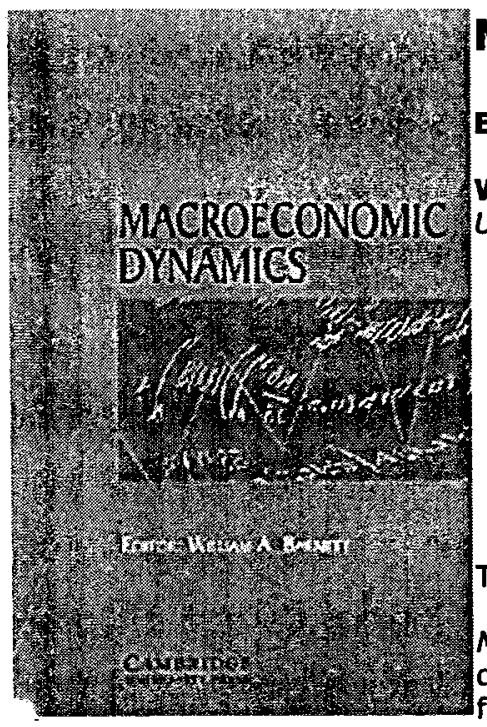

Macroeconomic Dynamics

Edited by

William A. Barnett

University of Kansas, USA

\author{
- Editorial Board Instructions for Contributors \\ - Pricing Full Text Online (purchase or subscribe) \\ - Links Advertising Rates
}

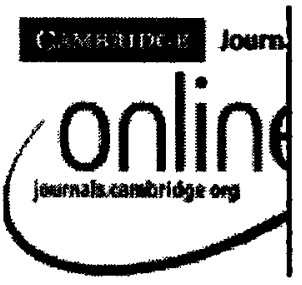

\title{
Current Issue
}

Volume 7-7, 2003

February, April, June September and Nove

To view a sample of this journal click here

Print ISSN: $1365-101$

Macroeconomic Dynamics publishes theoretical, empirical or quantitative research of the highest standard. Papers are welcomed from all areas of macroeconomics and from all parts of the world. Major advances in macroeconomics without immediate policy applications will also be accepted, if they show potential for application in the future. Occasional book reviews, software reviews, announcements, conference proceedings, special issues, interviews, dialogues, and surveys are also published. An electronic version of the journal is published simultaneously with the paper version, enabling immediate access to the best current research in macroeconomics. Forthcoming special issues include 'Robust Decision Theory and Policy Design in Economics' (M. Salmon, editor); 'Nonlinear Modeling of Multivariate Macroeconomic Relations' (P. H. Franses and T. Teravirta, guest editors); and 'Coordination, Learning, and Sunspots in Dynamic Models' (G. Evans and R. Guesnerie, guest editors).

Editor's journal page 
Home $>$ Journals $>$ Macroeconomic Dynamics

Introduction
Editorial Board
Instructions for
Contributors
Pricing
Full Text Online
(purchase/subscribe)
Links
Advertising Rates
Journals listed
alphabetically
Journals listed
by subject
Journals search

\section{Macroeconomic Dynamics}

Editor

William A. Barnett

Oswald Distinguished Professor of Macroeconomics Department of Economics

University of Kansas

Summerfield Hall, Room 213A

1300 Sunnyside Avenue

Lawrence, Kansas 66045-7585

barnett@ku.edu

\section{Book Review Editor}

Michele Boldrin

Department of Economics

University of Minnesota

1035 Managerial and Economics

Minneapolis, MN 55455

Professor Gregory D. Hess

Department of Economics

Bauer Center

Claremont McKenna College

500 E. 9th Street

Claremont, CA 91711

\section{Special Issues Editor}

Mark Salmon

Cass Business School, London, UK

\section{Editorial Information System Manager}

Steve Spear

Carnegie Mellon University, USA

\section{Advisory Editor}

Jean-Pascal Benassy

CEPREMAP, France

Willem Buiter

University of Cambridge, UK

\section{Guillermo Calvo}

University of Maryland, USA

v. V. Chari

Minnesota, USA

\section{Francis $X$. Diebold}

University of Pennsylvania, USA

http://www.ssc.upenn.edu/ diebold

Steven U. Durlauf

University of Wisconsin and Santa Fe Institute, USA

\section{Douglas Gale}

New York University, USA

\section{Giancarlo Gandolfo}

University of Rome, Italy

\section{Roger Guesnerie}

DELTA, France

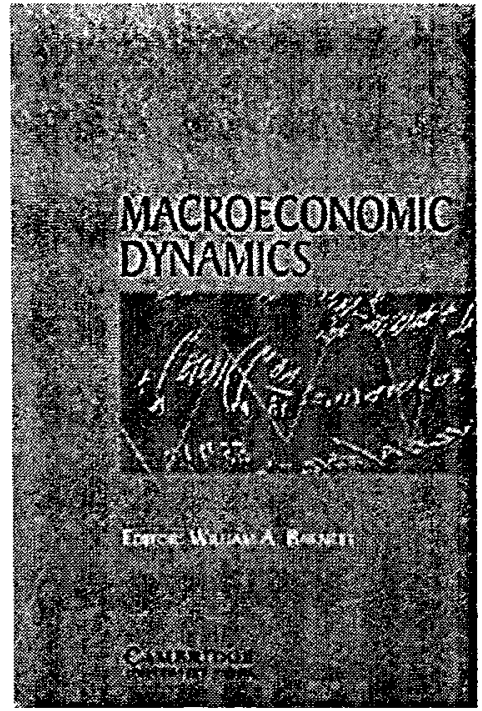

\section{Current Issue}

\section{Volume 7-7, 2003}

February, April, June, September and November

Print ISSN: $1365-1005$

Online ISSN: $1469-8056$ 
Takatoshi Ito

Hitotsubashi University, Japan

Timothy Kehoe

University of Minnesota, USA

Finn Kydland

Carnegie Mellon University, USA

Lung-Fei Lee

Ohio State University, USA

Iflee@econ.ohio-state.edu

Adrian Pagan

Australian National University, Australia

Peter Phillips

Yale University, USA

Julio Rotemberg

Harvard University, USA

Robert Townsend

University of Chicago, USA

Professor Mark Watson

Princeton Universoty, USA

Kenneth West

University of Wisconsin, USA

Michael Woodford

Princeton University, USA

Randall Wright

University of Pennsylvania, USA

\section{Associate Editor}

Masanao Aoki

UCLA, USA

Roland Benabou

Princeton University, USA

Professor Caroline M. Betts

University of Southern California, USA

Volker Boehm

University of Bielefeld, Germany

Dr Satyajit Chatterjee

Federal Reserve Bank of Philadelphia, USA

Professor Marcelle Chauvet

Federal Reserve Bank of Atlanta, UC Riverside, USA

Carl Chiarella

University of Technology Sydney, Australia

Professor Timothy Cogley

Arizona State University, USA

Professor Russell Cooper

Boston University, USA

Professor Isabel H. Correia

Universidade Catolica Portuguesa, Portugal

Professor Rose-Anne Dana

- France

Professor Jean-Pierre Danthine

University of Lausanne, Switzerland

Professor William Dupor

University of Pennsylvania, USA 
Janice Eberly

Northwestern University, USA

Alvaro Escribano

Universiday Carlos III de Madrid, Spain

Professor George Evans

University of Oregon, USA

Professor Roger Farmer

UCLA, USA

Professor Philip Hans Franses

Erasmus University, Rotterdam, The Netherlands

Professor Oded Galor

Hebrew University, Israel

Thorvaldur Gylfason

University of Iceland, Iceland

Melvin Hinich

University of Texas, USA

Professor Cars Hommes

University of Amsterdam, The Netherlands

Professor Seppo Honkapohja

University of Helsinki, Finland

Andrew Hughes Hallett

Cardiff University Business School, UK

Professor Svend Hylleberg

Aarhus University, Denmark

Patrick Kehoe

University of Pennsylvania, USA

Professor Pamela Labadie

George Washington University, USA

Professor Helmut Luetkepohl

European University Institute, Italy

Dr Pierre Malgrange

CEPREMAP, Paris, France

Dr David Marshall

Federal Reserve Bank of Chicago, USA

Professor Alfredo Medio

University of Venice, Italy

Professor Ilian Mihov

INSEAD, France

Professor Pablo Neumeyer

Universidad Torcuato Di Tella, Argentina

Professor Serena Ng

Johns Hopkins University, USA

Professor Lee Ohanian UCLA, USA

Simon Potter

Federal Reserve Bank of New York, USA

Dr Nell Rankin

University of Warwick, UK

Professor Lucrezia Reichlin

Free University of Brussells, Belgium

Professor Gilles Saint-Paul

Université des Sciences Sociales de Toulouse, France 
Professor Tony Smith

Carnegie Mellon University, USA

Professor Peter Norman Sorensen University of Copenhagen, Denmark

Professor Alan Sutherland

University of St Andrews, UK

Professor Stephen Turnovsky

University of Washington, USA

Wouter den Haan

University of California, San Dlego, USA

\section{Editorial Associate}

Melinda Barnett

University of Kansas, USA

Cambridge University Press 2001. Privacy Pollcy, Security. Jrder by phone $(+44(0) 1223326070)$ or fax $(+44(0) 1223326111)$. 


\section{CAnkngri: Jounals}

Home > Journals > Macroeconomic Dynamics > Table of Contents

Features

Related Journals

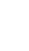

Journals

By Title

By Subject

Highlights

New Journals 2004

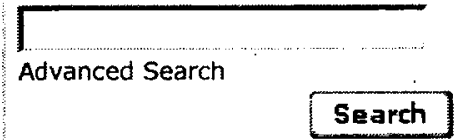

Free journal TOC alerts

New title information alerts

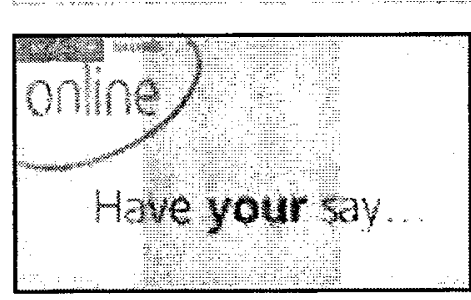

\section{Macroeconomic Dynamics}

Table of Contents (Volume 7, Issue 4)

Seasonality And Monetary Policy (pp 477 - 502)

Pere Gomis-Porqueras, Bruce D. Smith

Abstract

Heterogeneous Bellefs, Risk, And Learning In A Simple Asset-

Pricing Model With A Market Maker (pp 503 - 536)

Carl Chiarella, Xue-Zhong $\mathrm{He}$

Abstract

Cost-Of-Living Adjustments And Business Cycles:

Disaggregated Evidence (pp 537 - 566)

Magda Kandil

Abstract

Modeling High-Frequency Foreign Exchange Data Dynamics ( $p p$ $618-635)$

òscar JordÀ, Massimiliano Marcellino

Abstract

Testing The Expectations Theory of The Term Structure of Interest Rates In Threshold Models (pp 567 - 585)

Michael P. Clements, Ana Beatriz C. GalvÃo

Abstract

Education And Technology Adoption In A Small Open Economy: Theory And Evidence (pp 586 - 617)

Krishna B. Kumar

Abstract

Risk When Some States Are Low-Probability Events (pp 636 -

643)

Melvin J. Hinich

Abstract

Journal homepage
Editorial Board
Instructions for Contributors
Advertising Rates
Links
Subscriptions (1njtututionö
Print ISSN: $1365-1005$
Online ISSN: $1469-8056$
Online: $£ 150.00$
Print \& Onllne: $£ 168.00$
Print Only: $£ 150.00$
Full pricing details
Subscribe

\section{Table of Contents}

Current volume: $7: 1$ - 7:5,

All issues

\section{Sample Issue}

Vlew a free sample of this jo

(C) Cambridge Orer by phone 800-872-7423 (U.S. and Canada) 95-800-010-0200 (Mexico) or 914-937-9600, or by fax 914-937-4712.

All other countries: Order by phone $(+44(0) 1223326070)$ or fax $(+44(0) 1223325150)$ 


\section{|S I}

THOMSON SCIENTHE

(Use Browser Back button to return to previous page)

\section{ISI Master Journal List} JOURNAL LIST

Search terms: 1365-1005

Total journals found: 1

1. MACROECONOMIC DYNAMICS

Bimonthly

ISSN: $1365-1005$

CAMBRIDGE UNIV PRESS, 40 WEST 20TH ST, NEW YORK, USA, NY, 10011-4211 